\title{
Fat Body Phospholipid State Dictates Hunger Driven Feeding Behavior
}

Kevin P. Kelly¹, Mroj Alassaf*1, Camille E. Sullivan ${ }^{1}$, Ava E. Brent ${ }^{1}$, Zachary H. Goldberg ${ }^{1}$, Michelle E. Poling ${ }^{1}$, Julien Dubrulle ${ }^{2}$, Akhila Rajan"1

*Equal Contribution \#Corresponding and Lead Author: akhila@fredhutch.org

$5 \quad{ }^{1}$ Basic Sciences Division, Fred Hutch Cancer Research Center, Seattle, WA, USA

${ }^{2}$ Cellular Imaging Core, Shared Resources, Fred Hutch Cancer Research Center, Seattle, WA, USA

\section{Abstract}

Diet-induced obesity (DIO) leads to dysfunctional feeding behavior. But the precise molecular nodes that are dysregulated by DIO that alter satiety sensing and feeding motivation are not fully disentangled. The fruit fly is a simple genetic model system yet displays significant evolutionary conservation to mammalian nutrient sensing and energy balance. Using a longitudinal high sugar regime in Drosophila, we sought to address how lipid alteration in fat cells alters feeding motivation. We find that long-term exposure to an HSD increases baseline feeding in flies. However, prolonged exposure to HSD degrades the hunger-driven feeding (HDF) response.

15 Lipidomics analysis reveals that longitudinal exposure to HSD significantly alters whole body phospholipid profiles. Then, performing a systematic screen for phospholipid enzymes, we identify a specific enzyme, PECT, a rate-limiting enzyme in the phosphatidylethanolamine (PE) biosynthesis pathway and the fly ortholog of human PCYT2, was critical to maintaining hungerdriven feeding motivation. We show that disrupting PECT only in the fat body causes insulinresistant phenotypes and a loss of hunger-driven feeding. Excitingly, we find that overexpression of PECT restores HSD induced loss of hunger-driven feeding response. Strikingly human studies have noted a correlation between PCYT2/PECT levels and clinical obesity. Now, our unbiased studies in Drosophila provide specific genetic evidence for PECT in maintaining nutrient sensing during DIO. Our study provides novel insights on the role of phospholipids in interorgan communication of nutrient status. 


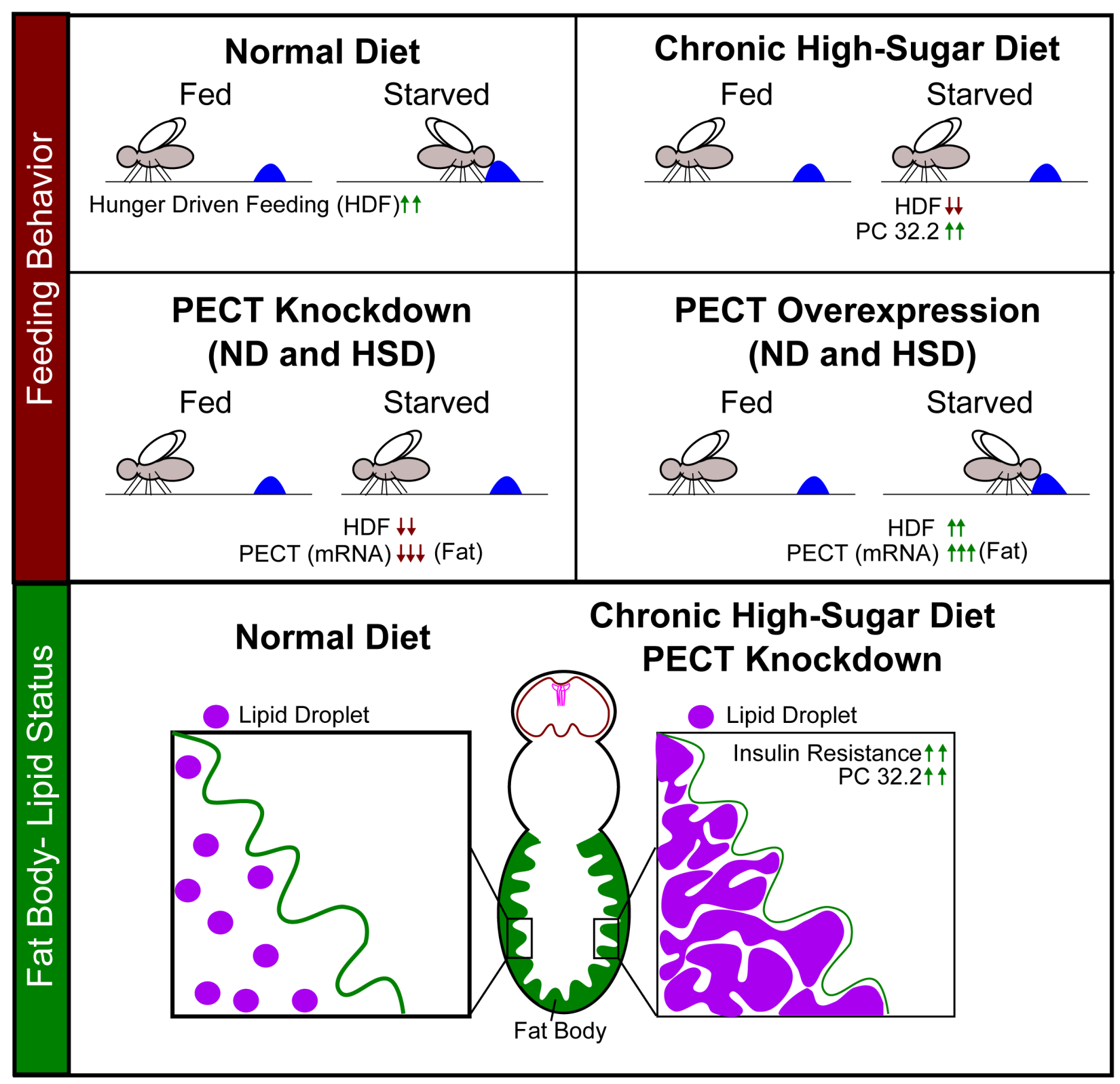




\section{Introduction}

High sugar diets and western diets in humans have been shown to impact feeding behavior and metabolism that leads to insulin and leptin resistance in mammals ${ }^{1-3}$. Leptin and insulin are endocrine factors that regulate systemic metabolism ${ }^{4-9}$. With interorgan signaling being a critical component to energy homeostasis in animals, composition of the diet itself may impact feeding behavior.

The components of diet induced obesity and insulin resistance are highly evolutionarily conserved in both flies and mammals ${ }^{10-14}$. Drosophila are an established metabolic model organism and have been shown to mimic feeding behavior of humans in response to high palatable foods ${ }^{15-19}$. There have been many studies on feeding behavior and appetite focused on the Drosophila brain identifying key neurons and receptors invovled ${ }^{20-22}$, although how peripheral organs communicate with the brain and how this communication is dysregulated in obesogenic diets is still unclear. Furthermore, Drosophila feeding behavior also has the advantage of being monitored at all stages of adult life in a short time span and does not require accounting for hoarding behavior seen in rodents ${ }^{23-27}$. Many base components of food such as free fatty acids and glucose have receptors that trigger major changes in cell transcription. While free fatty acids and diacyl/triglycerides have been well studied in feeding behavior, there is relatively little known about the impact of phospholipids on feeding behavior. Phospholipids can serve as signaling components as well. More importantly, phospholipids comprise the lipid bilayer of cells, and changes to its composition can alter the permissibility of the membrane ${ }^{28}$. Phospholipids also are key components required for endocytosis and trafficking of proteins for secretion. Altering phospholipid composition can disrupt secretion due to defects in vesicle formation ${ }^{29,30}$. Alterations in phospholipid composition have been identified in a number of obesity studies ${ }^{31-33}$. Furthermore, conserved enzymes involved in phospholipid homeostasis such as EAS (for PC) and PECT (for PE) have been shown to alter metabolism and biological processes impaired in diabetes such as cardiac function and neurodegeneration in flies ${ }^{34,35}$ and key phospholipid biosynthesis enzymes are correlated with obesity in human GWAS studies ${ }^{31}$. While obesity and changes to the lipidome are correlated, the functional relevance of these findings is still not well understood. Thus, it is possible that if prolonged changes to diet can impact phospholipid composition, defects in peripheral tissue to brain signaling could occur. Despite these intriguing possibilities, there have not been functional genetic studies that have systematically examined the effect of phospholipid metabolism and its dysregulation on feeding behavior. 
Studies by other groups have shed light on how chronic high sugar diet (HSD) results in both water imbalance and insulin resistance. Moreover, this study showed how HSD induced metabolic imbalance correlated with human physiology ${ }^{36}$, suggesting that using a chronic HSD feeding regime in adult flies will allow for the discovery of specific mechanisms relevant to human biology. With an established regime to induce obesity and insulin resistance through diet induced obesity in flies, we aimed to use a chronic HSD exposure model in adult Drosophila to identify evolutionarily conserved mechanisms by which HSD induced lipid imbalance, resulting in dysregulated nutrient sensing and feeding behavior.

In this study, we assess the feeding behavior of flies across their adult lifespan using a previously characterized method of eliciting diet induced obesity. We find wildtype flies display a distinct feeding behavior of significantly increased feeding upon starvation compared to fed controls, termed hunger driven feeding (HDF). Upon given high sugar diet (HSD) over their lifespan, flies gradually lose this starvation response. Lipidomics data revealed that large changes in phospholipid concentrations similar to data in human obesity clinical trials also occur in HSD fed flies at the time HDF was lost ${ }^{31-34}$. We further show that genetic modulation of the key phosphatidylethanolamine (PE) biosynthesis enzyme PECT in the fat body, which regulates Apolipoprotein based delivery of phospholipid species to the brain, is critical for regulating hungerdriven feeding. Specifically, decreasing PECT in the fat body leads to loss of HDF. Significantly, PECT overexpression in the fat body is sufficient to reverse the negative effect of HSD induced defects in hunger-driven feeding. These data suggest PE composition in the fat is a critical component of organismal feeding behavior and that chronic exposure to HSD disrupts PE homeostasis which in turn results in dysfunction of hunger-driven feeding. Our results point to a specific enzyme whose activity is sufficient to reverse the negative effect of chronic HSD on feeding behavior.

\section{Results}

\section{Hunger Driven Feeding in Flies is lost under High Sugar Diet}

The inability to sense hunger underlies a multitude of eating disorders, including obesity ${ }^{37}$. Yet, the cellular and molecular mechanisms governing the breakdown of the hunger-sensing system in diet-induced obesity are unknown. To address this, we use a Drosophila obesity model. 
To first determine if flies were a suitable candidate to measure hunger-driven feeding behavior and to identify critical timepoints for hunger-sensing breakdown, we first quantified feeding behavior in flies under their normal diet conditions (Figure 1). Figure 1A depicts the experimental method for monitoring fly feeding activity over time on different diets. In brief, flies were housed in vials of normal diet for 7 days after eclosion. This was then followed by maintain flies on the treatment diet throughout the fly's adult life. 16 hours prior to measurement, half the flies that were to be monitored were moved to a $1 \%$ sucrose/agar media, which is a minimal food media to elicit a starvation response (herby referred to as Stv condition). Flies were then monitored for feeding on liquid diet using a Fly Liquid Interaction Counter (FLIC) for three hours (10am-1pm local time). Under normal diet conditions, wild type (W1118) flies showed significantly higher feeding when placed under 16 hours of starvation media than if allowed to eat ad libitum, consistent with a hunger driven feeding (HDF) response also seen in vertebrates (Figure 1B.) ${ }^{38,39}$. To determine if age had an impact on HDF, adult flies were measure at multiple stages of their lifespan. Throughout the fly's adult lifespan, flies significantly increased their feeding after a 16 hour starvation (Figure 1B).

To see if hunger driven feeding behavior is altered by an obesity-inducing diet, flies were placed on a High Sugar Diet (HSD) containing 60\% more sucrose than normal media and measured fly HDF over the adult fly lifespan (Figure 1C). Flies fed an HSD displayed a distinct starvation response in their early adult stages with significant increases in feeding upon starvation. However, continued exposure to HSD over time begins to weaken the response to starvation, with flies showing no significant change in feeding behavior between fed and starvation conditions by 14 days of HSD ad libitum feeding suggesting a breakdown in the hunger-sensing system. Notably, flies fed a HSD showed either similar or significantly higher basal feeding activity comparted to flies fed a normal diet. This suggested that, despite the increased caloric density of the HSD, hunger-sensing is disrupted (Figure S1). Taken together, this data shows that flies on HSD both eat more yet lose their responsiveness to metabolic challenges after chronic exposure.

\section{Insulin Resistance and Lipid morphology changes at point of HDF loss}

The loss of hunger driven feeding behavior after 14 days of HSD suggested this was a critical timepoint of metabolic disruption. Given that insulin signaling is a major regulator of systemic metabolism, and insulin resistance is a hallmark of obesity, we asked whether flies fed a HSD had dysfunctional insulin signaling. To answer this, we first measured the amount of 
Drosophila insulin-like peptide (Dilp) accumulation in Insulin Producing Cells in the brain (IPCs, Figure 2A). We found that 14 days of HSD feeding ad libitum resulted in a significant decrease in Dilp accumulation in the IPCs, suggesting increased Dilp secretion in the HSD fed flies. Because of the increase in insulin signaling, we predicted that insulin resistance may be occurring at this time. To determine this, we measured the forkhead box O (FOXO) nuclear localization in the fat body (Figure 2B). Insulin signaling is activated by the binding of insulin to its cell surface receptor. When activated, IR auto-phosphorylates, which triggers the phosphorylation of multiple downstream targets including the transcription factor FOXO. Phosphorylation of FOXO prevents it from entering the nucleus and initiating the gluconeogenic pathway, a starvation response pathway. Thus, FOXO nuclear localization has been used as a proxy to monitor insulin sensitivity 40,41. FOXO was found to be significantly more localized in the nucleus in flies fed HSD for 14 days compared to their normal diet counterparts. Notably, this effect was not seen after an acute sixhour exposure to HSD, suggesting this impact on insulin resistance only occurs after chronic exposure (Figure S2B). Because insulin secretion is higher in the HSD group yet shows more FOXO nuclear localization, this suggests a decrease in insulin sensitivity in the fat body of 14 day HSD fed flies.

Accumulation and enlargement of lipid droplets, the cell's lipid storage organelles, are associated with insulin resistance ${ }^{42}$. Therefore, we wanted to investigate the effects of HSD of lipid droplet morphology in the fat body of flies over time. At 7, 14, and 21 days, flies on HSD showed progressively larger, misshapen, and more dense lipid droplets compared to flies on ND (Figure 2C). Together, these data suggest that defects in HDF are associated with insulin resistance in the fat body.

\section{PE and PC are elevated at point of HDF loss}

Because of the disruptions to fat body insulin response and morphology, we sought to profile the lipidome of flies on normal fed versus HSD. W1118 flies fed Normal diet or HSD were assessed for changes in lipid classes using a targeted quantitative lipidyzer after 7 and 14 days on their respective diets. When normalized to the normal diet flies, HSD flies after 7 days show significant elevation of free fatty acids (FFAs), triacyl glycerides (TAGs), and diacyl glycerides (DAGs) (Figure 3A, left) which is consistent with other studies ${ }^{13}$. After 14 days, the difference in

FFAs, TAGs, and DAGs was further increased, suggesting a compound effect of the HSD as well as an overall increase in lipid content in the HSD flies. However, FFAs, TAGs, and DAGs were not the 
only lipid classes altered by day 14 in HSD. Indeed, almost all lipid classes except for ceramides (CE). In particular, there was a significant increase in the phospholipids phosphatidylethanolamine (PE, Figure $3 \mathrm{~A}$ and $3 \mathrm{~B}$ left) and phosphatidylcholine (PC, Figure $3 \mathrm{~A}$ and 3B right). Notably, lysophosphatidylethanolamine (LPE) and lysophosophatidylcholine (LPC) which can be converted to PC and PE were significantly decreased in the HSD group compared to the normal fed (NF) group. Phospholipids make up the lipid bilayer of the cell's membrane, as well as secretory vesicles ${ }^{43}$, and disruption of either is able to impact signaling ${ }^{44}$.

\section{4-day HSD induces significant changes in PC and PE class levels and properties}

To understand how phospholipid levels were impacted by HSD, we further assessed the phospholipid lipidomic data from Figure 3 to the class and species level (Figure 4 and Figure S4 for a general overview of phospholipid composition). Significant increases to PC32.2 (p<0.00001) and $32.0(p=0.025)$, which are lipid classes found in previous studies to be influenced by PECT (Figure 4A.) $)^{35}$. At day 7, when PE and PC overall concentration were unaltered, lipid classes were not significantly altered (Figure 4A). By day 14, there were significant increases PC32.2 and 32.0 at the class level (Figure S4B) as well as an overall elevation for most species in the PE34.1, PE36.2, PC32.2, and PC32.0 classes with several species significantly elevated compared to the NF group. Taken together, this data suggested a global increase in PE biosynthesis in the fly through the PECT-mediate PE biosynthesis pathway at the time of HDF loss.

Because lipid membrane fluidity is an important component of signaling at both cell to cell and tissue to tissue level, we measured changes at the species level for PE (Figure 4B) and PC (Figure 4C) based on the number of doublebonds present. Doublebonds create kinks in the lipid bilayer, leading to increased lipid membrane fluidity which impacts vesicle budding, endocytosis, and molecular transport. At day 7, there was no significant difference in the number of double bonds species for PC but there were significantly higher concentrations of PE species with 3 or 4 doublebonds for the HSD fed flies. By day 14, a dramatic shift in species based on doublebond numbers is seen for both PC and PE. While both show a significant increase in species with no doublebonds, the largest changes in concentration are seen for species with 2-4 doublebonds (4B and C). Taken together, this data suggests that HSD results in an increase in specific PC and PE lipid classes, thus creating a more fluid lipid bilayer environment that likely impacts signaling dynamics.

\section{Apolipoprotein accumulation in the brain is altered by fat body PECT expression}


While HSD is likely to affect lipid levels in every cell type, the PC and PE lipid classes that showed major alterations are primarily synthesized by the fat body and trafficked in lipophorin particles to other organs including the brain. Drosophila has two major lipophorin chaperones ApoLTP and Apolpp. Apolpp is the functional ortholog of human ApoB ${ }^{45}$. Palm et al. have demonstrated that knock down of Apolpp from fat results in a significant reduction in circulating $\mathrm{PE}$ and $\mathrm{PC}$ species in the hemolymph ${ }^{45}$. We noted that the PE and PC species that showed specific alterations on HDF, especially PC 32.2 (see discussion), was reminiscent of a known don of the PE enzyme PECT. To see if Apolpp accumulation could be altered by changes in phospholipid production, we measured Apolpp puncta and percent area covered of IPCs covered by Apolpp in 14-day old adult flies with fat body-specific knockdowns of Luciferase (Figure 5A, top), easily shocked (EAS), a PE biosynthesis protein that phosphorylates ethanolamine (Figure 5A, middle), or PECT (Figure 5A, bottom). This measurement was performed using an Apolpp antibody developed in the lab (validation shown in Figure S2A). We found that compared to the luciferase knockdown control, a knockdown of EAS did not show an impact to Apolpp levels in the IPC. Conversely, a knockdown of PECT in the fat body caused a significant decrease in Apolpp levels in the IPC. PECT expression was measured via qPCR to and confirmed to be knockdown in these flies (Figure S3B, left). To see if overexpression could have the opposite effect, we then measured brain apolipoprotein in 14-day old flies with fat body specific overexpression of Luciferase (Figure 5B, top) and PECT (Figure 5B, bottom). Indeed, we find that PECT overexpression in the fat body 20 led to significantly higher levels of Apolpp compared to the luciferase control. PECT overexpression flies were also confirmed to have elevated PECT expression via qPCR (Figure S3B, right). Given how PC32.2 is elevated after 14 days of exposure to HSD, we assumed that PECT levels would be high for flies under HSD. Surprisingly, at day 7 and 14, PECT expression is significantly higher in HSD fed flies compared to NF. Because the elevation of PC32.2 in HSD indicate a PECT loss of function or downregulation, the qPCR data likely show an upregulation in an attempt to mediate the PECT dysregulation occurring at the protein level. Taken together, these results show manipulation of PE biosynthesis through PECT is able modulate the amount of apolipoprotein released by the fat body to the brain. These changes to PECT also seem to be occurring during HSD feeding, with HSD fed flies being in an PECT overexpressed state during 14 days of HSD feeding.

PECT knockdown shows FOXO accumulation and lipid morphology changes similar to HSD 
Given that PE homeostasis is disrupted under HSD (Figure 3), and that coincides with insulin resistance, we asked whether PECT activity in the fat body is important for maintaining insulin sensitivity. To address this, we compared the levels of nuclear FOXO of 14-day old fat body specific PECT knockdown flies (Figure 6A, right panel) to their luciferase knockdown counterparts (Figure 6A, left panel). Much like the results after 14 days of HSD in wildtype flies (Figure 2B), we found significantly higher localization of FOXO in the nucleus of the PECT knockdown flies than the control (Figure 6A, graph). Given that lipid droplets serve as a repository for lipids, the building blocks of phospholipids, we reasoned that knocking down PECT will lead to excessive accumulation of lipids and result in enlarged lipid droplets resembling flies on HSD. Indeed, a qualitative view of lipid droplets in PECT knockdown flies showed larger and more clustered lipid droplets than control. This was similar to the effects of HSD, although both the control and the PECT knockdown in this experiment were on normal diet. Given that knocking down PECT in the fat body was enough to replicate FOXO and lipid droplet phenotypes observed in flies under HSD, this suggests that PECT plays an important role in mediating insulin sensitivity in the fat body.

\section{PECT knockdown in the fat modulates hunger driven feeding behavior}

Since disrupting PE biosynthesis via PECT knockdown in the fat body resulted in similar molecular and morphological changes to the fat bodies of flies on HSD, we sought to determine if a fat body specific manipulation of PECT was enough to modulate hunger driven feeding behavior in flies. To do this, we monitored feeding behavior of adult flies with a fat body specific knockdown of either luciferase (control), EAS, Pcyt2 (a key PC biosynthesis enzyme), or PECT given either normal diet or HSD for 14 days (Figure 7A). The luciferase knockdown control flies displayed strong hunger driven feeding in both NF and HSD groups by day 14 of their diets, showing a large increase in feeding upon 16-hour starvation. When impacting the PC pathway through Pcyt2 knockdown, HDF was unaffected showing a comparable increase in feeding on starvation as the control. This suggested that while PC biosynthesis was altered in HSD, a fat body disruption of PC was not responsible for the HDF behavioral changes seen. A Knockdown of EAS also showed a hunger driven feeding response in the NF group, however it did lose HDF when flies were given HDF. Finally, a knockdown of PECT showed a complete loss of HDF when flies were fed both normal diet and HSD, showing no significant change in feeding behavior in response to starvation (red outline). Interestingly, there are two major pathways of PE biosynthesis, with PECT being the rate-limiting component of the ER-mediated PE biosynthesis pathway and PISD being the rate-limiting enzyme in the mitochondria mediated PE biosynthesis pathway. We also knocked 
down PISD in the fat body and found that it showed similar disruption to HDF as the PECT knockdown, suggesting that disrupting PE biosynthesis either through the ER-mediated or the mitochondrial-mediated pathway was enough to impact feeding behavior (Figure S2A). When overexpressing PECT in the fat body, HDF returns in both normal diet and HSD fed flies (Figure 7B).

5 Furthermore, by overexpressing PECT, flies maintain their starvation response after 14 day of HSD exposure where the loss of starvation response was found to occur for wildtype flies, suggesting overexpression provides a gain of function to maintain HDF (Figure 1C and 7B). This effect is maintained after both 7 days and 14 days on HSD treatment, where 14 days of HSD caused a loss of HDF in W1118 flies. In conclusion, PE biosynthesis in the fat body is an important component for feeding behavior in flies, can be modulated by diet, and effect signaling and communication of the fat body to other peripheral tissues. 


\section{Discussion}

The Drosophila model organism has been shown to be a relevant model for mimicking the conditions of human diet induced obesity and insulin resistance ${ }^{15}$. Studies from Dus and Ja et al. have previously performed measurements on taste preference, feeding behavior/intake, survival, etc. using a high sugar diet diet-induced obesity model and have found much in common with their mammalian counterparts. However, no study has longitudinally monitored feeding behavior under this model, with the longest measurement of adult feeding behavior being around 7 days after eclosion. Because the mammalian model of insulin resistance is complicated due to the complexity of other secreted hormones and proteins involved, the Drosophila DIO model is a yet untapped model to assess evolutionarily conserved traits of chronic DIO exposure in a simpler system.

To determine if this DIO Drosophila would be useful for understanding interorgan communication, we first characterized feeding behavior. Changes in feeding behavior in both vertebrates and invertebrates is thought to occur via communication of peripheral organs responsible for digestion/energy storage and the brain ${ }^{46}$. This communication is performed by the peripheral tissues secreting hormones or other factors to the brain that provide information on nutritional state ${ }^{46}$. One example of such an interaction is leptin signaling, which is released from the adipose tissue and elicits insulin signaling from the brain, promoting satiety and lack of appetite ${ }^{47,48}$. Leptin has long been studied as an obesity/satiety hormone, however work on leptin in mice and the homolog Upd2 in flies suggest that a key function is to respond to starvation response $e^{5,47,49,50}$. Our lab found that exposing flies to HSD resets synaptic contacts between Leptin/Upd2 sensing neurons and Insulin neurons ${ }^{49}$. In an effort to delineate how else HSD may alter systemic communication between fat-brain systematically analysed when hunger-driven feeding behavior is disrupted by HSD and how this correlates with $\mathrm{ft}$ body lipid status. Our study finds that under normal diet conditions, flies display a clear response to starvation in the form of elevated feeding immediately following a starvation period, when compared to ad libitum counterparts irrespective of age. When flies are placed on the obesity inducing diet, the baseline feeding behavior is always higher than NF control sibs at any age (we measured up to 28 days). However, the response of HSD flies to Hunger Driven Feeding (HDF) is present after a short period of exposure, but is lost after a chronic exposure of 14 days. While feeding behavior measurements have not been measure past 10 days, our acute data is in line with previous literature as we do note an increase in baseline feeding in HSD after acute exposure which has been previously 
reported ${ }^{17,50}$. By 14 days of HSD, we find flies lose their HDF and show a similar response to food as ad libitum fed. Our data indicate that if flies are exposed to a DIO for a prolonged period, a fundamental change to the brain causing the loss of HDF. Furthermore, a measurement of the Insulin (Dilp) neurons and fly fat at the time of loss HDF show evidence of insulin resistance. It should be noted that previous work from Dam et al. have established that under our HSD conditions, there is a water imbalance that is associated with decreased survival and $\mathrm{pH}$ imbalance ${ }^{36}$. While the impact of insulin resistance was proven to be a process independent of the water imbalance, it will be important for future studies to consider if the feeding behaviors observed in this study are altered given a water supplement. This change in feeding behavior and appetite is the same seen in diabetics and overweight human studies, suggesting this could be the same change in behavior brought on by insulin resistance in humans.

Since this physiological change occurred after 14 days on HSD, we then sought to determine what caused this drastic change at this time. First, we analyzed the lipid profile as the diet would likely cause disruptions. As expected, a 7-day exposure to HSD did elevate overall content of neutral lipids compared to normal fed counterparts, with TAGs and DAGs being the most increased. This has previously been reported in this model and similarly consistent with other DIO models ${ }^{13,17,36}$. At 14 days, the time of HDF loss, this elevation of TAGs and DAGs persists but we now observed disruptions to other lipid classes, particularly increases in Phosphatidylethanolamine (PE) and Phosphatidylcholine (PC) and decreases in lysophosphatidylethanolamine (LPE) and lysophosphatidylcholine (LPC). Excitingly, these changes in lipid classes have also been seen in diabetics and individual predisposed to obesity and their involvement in insulin resistance is clear but not functionally characterized ${ }^{31-33}$. While this study focused on the role of PC and PE biosynthesis, the fact that Drosophila also displays a distinct drop in LPE and LPC should also be explored. It will be interesting to determine if mutating genes responsible for PC to LPC or PE to LPE pathways are also able to impact behavior or if these effects are independent of LPC/LPE. Another striking finding was a general analysis of the phospholipid species showed higher concentration based on doublebond number. This has implications for overall signaling, as the higher prevalence of doublebonds allows for more kinks in the lipid membrane and likely is increasing the membrane fluidity ${ }^{29,30,44}$. While this was beyond the 30 scope of this study, it would be interesting to see how the fluid dynamics and vesicle formation have been altered by this change in fluidity at the time of HDF loss. Furthermore, analysis at the species level indicated that many of the species and classes increased were related to PECT 
(enzyme with same function as PCYT2 in humans). Because PCYT2 was previously identified in a separate GWAS study to correlate with BMI in humans, we chose to investigate the phospholipid biosynthesis pathway further.

To determine if PECT was a major component responsible for the loss of HDF, we first characterized the effects of knocking down PECT in the fat body. PECT molecular null mutation allele LL06325) results in lethality, and the PECT molecular null allele has been analysed in a mosaic tissue context in Drosophila photoreceptors to uncover its role in synaptic vesicle pool maintenance ${ }^{35}$. Given that the PECT null allele $\left(p e c t^{\mathrm{LL06325}}\right)$ is lethal, and mosaic analysis is incompatible with studying effects on whole animal physiology, we have relied on fat tissuespecific knockdown of PECT, using RNAi, to study its effects on feeding behavior. We find that when PECT is knockdown in the fat body, flies show similar signs of insulin resistance as the DIO model, despite eating normal diet. Our lipidomic data implied that signaling from fat to the brain may be perturbed. Indeed, in a fat body specific PECT knockdown, we see a decrease in Apolpp puncta in the brain. Apolipoprotein is the main vehicle that traffics lipids, specifically DAG and PE, from fat to all peripheral tissues including brain ${ }^{45}$. This makes it likely that Apolpp-carrying lipid particles are perturbed when PE biosynthesis is disrupted. Consistent with this hypothesis, we observed reduced Apolpp puncta in PECT knockdown (Figure 5A) and elevated puncta in the PECT over-expression (Figure 5B) in the Insulin neuron region. It has been shown by Eaton and colleagues that lipophorin signals regulate insulin neurons ${ }^{51}$. However, it is currently unclear if Apolpp is the direct regulator of feeding or whether it is ferrying signaling molecules along with PE/PC lipids. In future studies, an experiment to test this would be to isolate lipid vesicles generated by wildtype and PECT knockdown flies to determine what key signaling proteins are being altered in abundance. Most importantly, flies with PECT knockdown do not display hungerdriven feeding even on normal diet by day 14 . This change seemed specific to the PE biosynthesis pathway, as PISD, the critical enzyme for mitochondrial PE biosynthesis in flies, showed a similar impact on HDF as PECT, the critical enzyme for ER mediated PE biosynthesis. However, we did not observe this effect with Pcyt, the key enzyme for PC biosynthesis. When we manipulated EAS, a non-rate limiting component of PE biosynthesis, showed a decreased effect compared to PECT, only showing changes in HDF in the normal fed. This suggests only a major disruption in PE 30 biosynthesis is required to create the impacts seen in chronic HSD feeding. Excitingly, if PECT expression was elevated through a fat bod specific overexpression, we rescued HDF in normal diet 
fed flies at day 14 and were able to detect HDF at day 14 in the DIO flies, which was longer than seen under wildtype conditions.

In conclusion, we find in the Drosophila model that chronic DIO exposure creates a large change in phospholipid profile at times of insulin resistance and changes in starvation response. We have associated these changes in DIO behavior with decreases in PE biosynthesis and can be similarly produced by manipulating PECT. Overexpression of PECT was able to rescue and even prolong the animal's starvation response under DIO. While this is beyond the scope of this study, it will be interesting to know if similar disruptions to PCYT2 in mammalian adipose tissue or liver can deliver a similar effect on insulin resistance and if overexpression of mammalian PCYT2 could improve insulin resistance in DIO.

\section{Methods}

\section{Animals used and Rearing conditions}

The following strains were used in this manuscript: W1118, PISD-RNAi (Bloomington \#67763), PECT-RNAi (Bloomington \#67765), EAS-RNAi (Bloomington \#38528), Pcyt2-RNAi (Bloomington \#67764), Lpp-Gal4 on X (P.Leopold/ S. Eaton via Patrick J.52),Luciferase-RNAi (JF01355), and w;;UAS-PECT III (Flybase ID:FBal0347227, generously donated by Clandinin lab), UAS-HAApolopp-myc ${ }^{56}$. Flies were housed in $25^{\circ} \mathrm{C}$ incubators. In all experiments, only adult male flies were used. Flies were sexed upon eclosion and place on normal diet, a standard diet containing $15 \mathrm{~g}$ yeast, $8.6 \mathrm{~g}$ soy flour, $63 \mathrm{~g}$ corn flour, $5 \mathrm{~g}$ agar, $5 \mathrm{~g}$ malt, $74 \mathrm{~mL}$ corn syrup per liter, for 7 days. Anesthesia using a $\mathrm{CO} 2$ bubbler was used for initial sexing, then never used for the remainder of the experiment. After 7 days, flies were either maintained on normal diet or moved to a High sugar diet (HSD), composed of the same composition as normal diet but with $30 \%$ more sucrose, for the length specified in the figures (typically 7 or 14 days). For measurements of hunger driven feeding, a portion of flies from each diet were placed on starvation media (1\% Sucrose/1\% agar) for 16 hours prior to the experiment.

\section{Dilp5 and FOXO Imaging}

Fluorescent intensity of Dilp 5 in the IPCs was measured using Z-stack summation projections that included the full depth of the neurons were generated. A region of interest around the Dilp neurons 
was manually drawn and the integrated density values were acquired. To measure nuclear FOXO accumulation, a similar number of confocal stacks were acquired for each tissue sample.

\section{Puncta Quantification}

Quantification of the number of puncta and percentage area occupied by Apoll

5 immunofluorescence was done using ImageJ. Maximum-intensity projections of z-stacks that spanned the entire depth of the Dilp neurons at 0.3 um intervals were generated. A region of interest was manually drawn around the Dilp neurons and a binary mask for the Apoll channel was created using automated Moments thresholding values, which was followed by watershed postprocessing to separate particles. The number of particles and the area fraction were measured using the "analyze particles" function.

\section{Image analysis}

Image analysis was performed in the MATLAB R2020b environment, and the associated scripts are available at github address. FOXO accumulation in Drosophila adult fat cells was assessed by measuring the mean voxel GFP intensity in the nucleus that is delimited by the lamin antibody signal. To generate the 3D nuclear masks, Lamin stacks were first maximally projected along the zaxis, and after global thresholding, basic morphological operations and watershed transforms, the locations of the nuclear centroids in the $x-y$ plane were used to scan the z-stacks and reconstruct the nuclear volume. The accuracy of the segmentation was assessed by manual inspection of random cells. Once the nuclear compartment was reconstructed in $3 \mathrm{D}$, it was used as a volumetric mask to extract intensity values of the FOXO reporter signal, and to compute the mean voxel intensity in the nucleus. Two-sided Wilcoxon rank sum tests were performed to assess the statistical significance of pairwise comparisons between experimental conditions.

\section{Lipidomics}

Whole adult male flies were flash frozen in liquid nitrogen either after 7 or 14 days on normal diet or HSD. 10 flies were used per biological sample and 10 biological replicates were used for each diet and timepoint. Frozen samples were sent to the Northwest Metabolomics Research Center for targeted quantitative lipid profiling using the Sciex 5500 lipidyzer (see Hanson et al. for detailed methods $\left.{ }^{53}\right)$.

\section{Feeding Behavior}


For hunger driven feeding analysis, age-matched W1118 flies were given normal diet or HSD for 5, $7,14,21,24$, and 28 days after and initial 7 days of development on normal diet. All other experiments were performed for 7-day or 14-day durations. 16 hours prior to feeding behavior assessment, half of flies from each treatment were moved to starvation media. Individual flies were placed in a single well of fly liquid-food interaction counter (FLIC) and supplied with a 1\% sucrose liquid diet. Detailed methods for how FLIC operates can be found in Ro et al., 2014 ${ }^{23}$. Fly feeding was measured for the first three hours in the FLIC and all FLICs were performed at 10am local time. For each FLIC, half of the wells ( $n=6 / F L I C)$ contained the fed group and the other half contained the starved group of flies for direct comparison. 12-30 flies were measured for analysis of feeding and feeding was normalized to the control fed group as a percentage. A signal above 40 (a.u.) was considered a feeding event. Analysis of feeding events was performed using R.

\section{Gene Expression}

30 fly fat bodies of each genotype were dissected in RNAlater after 7 and 14 days on normal diet or HSD. Immediately after dissection, fat bodies were moved to tubes of 200uL RNAlater on ice. RNAlater was then removed, 30uL of trireagent and a scoop of beads were added, and fat bodies were homogenized using a bullet blender. RNA was then isolated using a Direct-zol RNA microprep kit following manufacturer's instructions. Isolated RNA was synthesized into cDNA using the BioRad iScript RT supermix for RT-qPCR and qPCR was performed using the BioRad ssoAdvanced SYBR green master mix. Primers used in the paper include Robl (control gene used for normalization) and PECT. Relative quantification of mRNA was performed using the comparative CT method and normalized to Robl mRNA expression. For each experiment, three biological replicates were used with 3 technical replicates used for qPCR.

\section{Statistical Methods}

t-test or ANOVA were performed using PRISM. Boxplots and standard deviation calculations were

25 either performed through prism or through R. R packages used in this paper included tidyverse, ggplot2, and ggthemes. 
1A. Hunger Driven Feeding Experimental Design

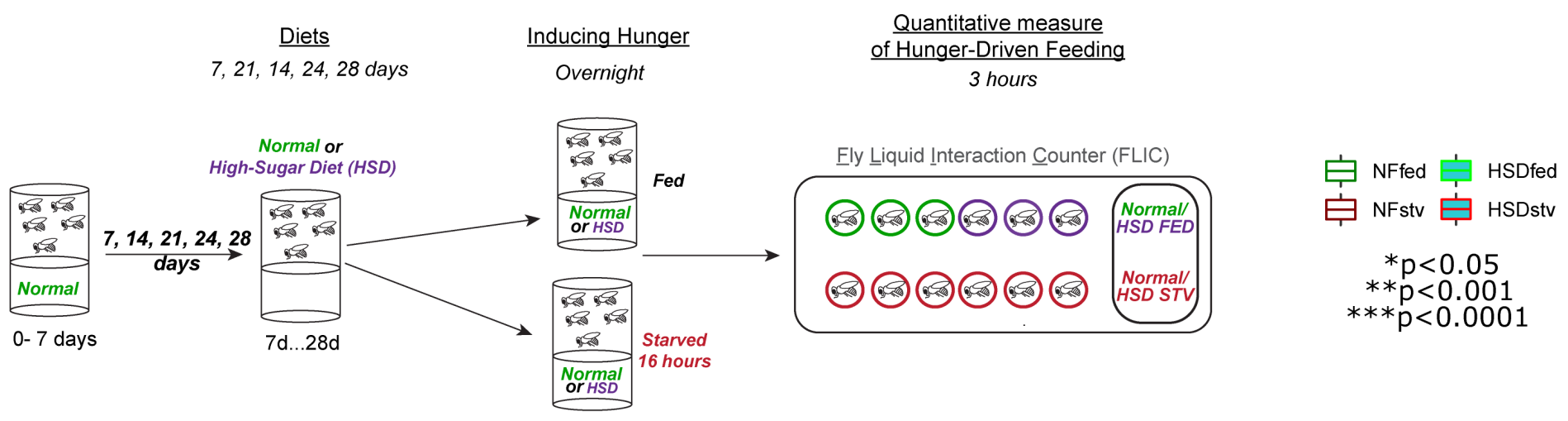

1B. W1118 Hunger Driven Feeding Response on Normal Diet (NF)
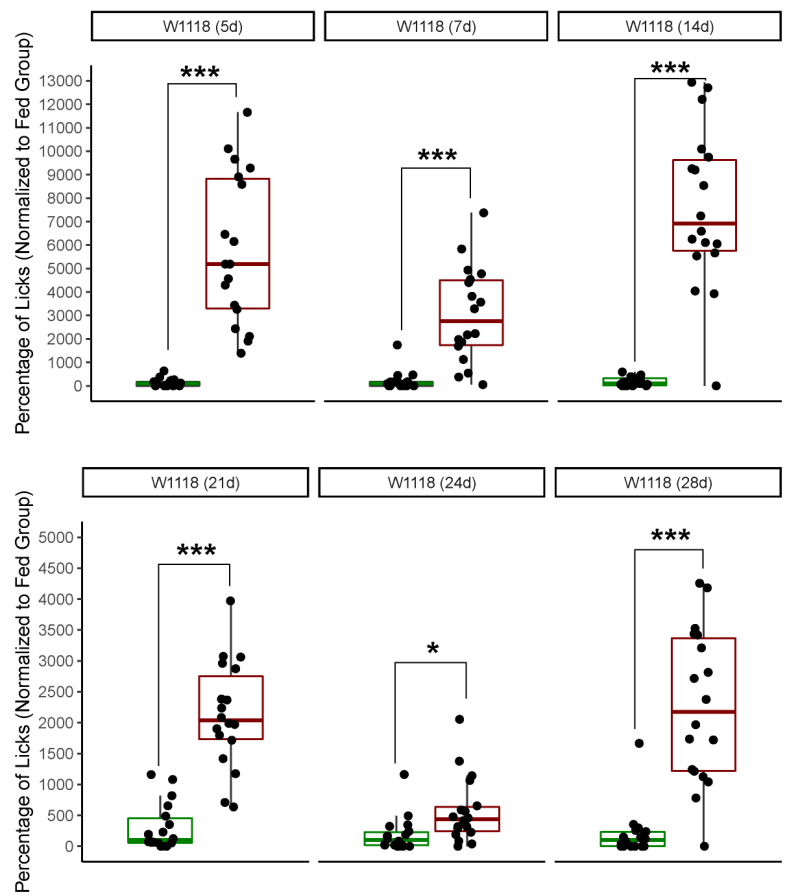

1C. W1118 Hunger Driven Feeding Response on High Sugar Diet (HSD)

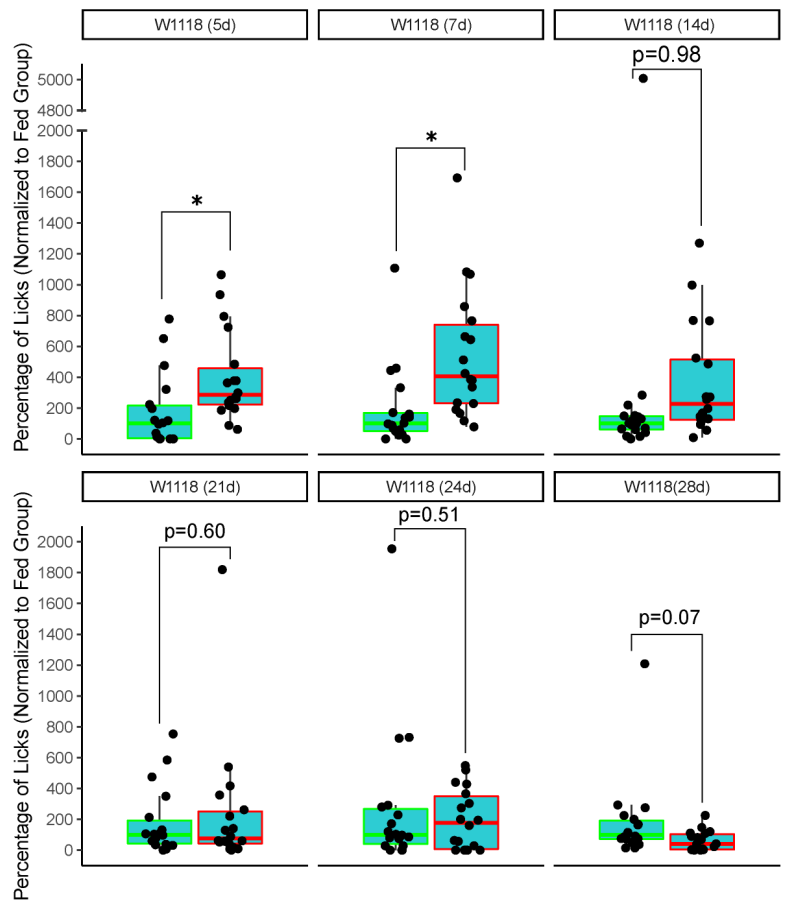




\section{Figure 1. W1118 Flies show loss of Hunger driven Feeding on HSD over time.}

Hunger driven feeding behavior in flies was tested using the schematic in A). After aging flies for 7 days on normal lab food, flies we subject to normal diet or high-sugar diet $(30 \%$ more sugar in

5 food) for a duration of 7 days-28 days. For every 7 days, hunger was induced by subjecting flies to starvation (agarose) overnight. Then quantitative feeding behavior was monitored in flies subject to normal food or high-sugar diets using the Fly liquid interaction counter. Feeding events were calculated and \% fold change in hunger-driven feeding is measure reported below $1 \mathrm{~B}$ and $1 \mathrm{C}$. Boxplots of fly feeding events on B) normal diet (NF, white fill) or C) High Sugar Diet (HSD, blue fill) at $5,7,14,21,24$, and 28 days on diet conditions ( $n=18 /$ diet/fed state/day). In W1118, flies experience a significant increase in feeding upon starvation challenge which is maintained throughout adult life. However, W1118 flies on HSD over time lose this increase in feeding after 14 days of HSD ad libitum feeding. Black dots represent and individual flies feeding. Freshly eclosed flies were first put onto NF diet for 7 days as they matured then either maintained on NF (B) or transferred to HSD media for the duration of the experiment. 16 hours prior to feeding measurements, flies were flipped either to fresh media (Fed group, green outline) or moved to a 1\% sucrose/agar media (Starved (Stv) group, red outline). Feeding events were automatically recorded for individual flies using a Fly Liquid Interaction Counter (FLIC). Total feeding events for the first three hours in the FLIC were tallied for each fly and the respective fed and starved groups were normalized as a percentage of feeding events, with the median fly feeding events for the fed group being considered as $100 \%$ feeding. Both fed and starved counterparts were measured in parallel on the same day. Asterisks indicate significance with ${ }^{*} p$ value $<0.01$, ${ }^{* *} p$ value $<0.001$, and *** $p$ value $<0.0001$. 
bioRxiv preprint doi: https://doi.org/10.1101/2021.12.16.472854; this version posted December 21,2021 . The copyright holder for this preprint (which was not certified by peer review) is the author/funder, who has granted bioRxiv a license to display the preprint in perpetuity. It is made available under aCC-BY-NC-ND 4.0 International license.

2A. Dilp accumulation in IPC after 14 days on NF/HSD Diet
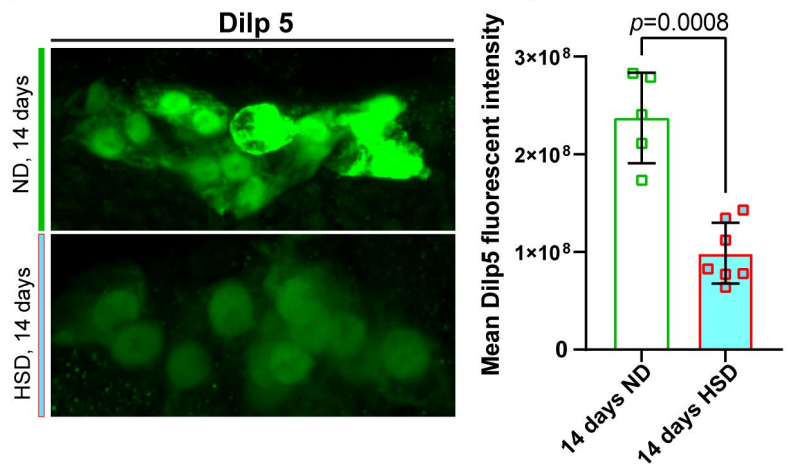

2B. FOXO Nuclear Localization after 14 days on NF/HSD Diet
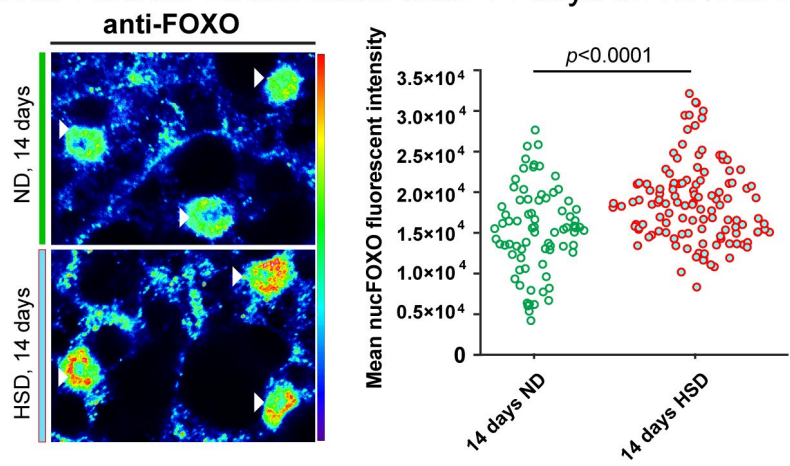

2C. W1118 Lipid Droplet Morphology on NF and HSD

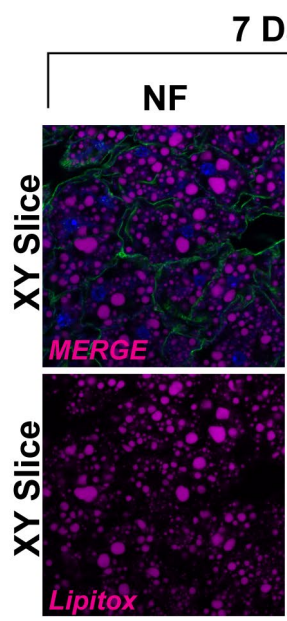

Days

14 Days
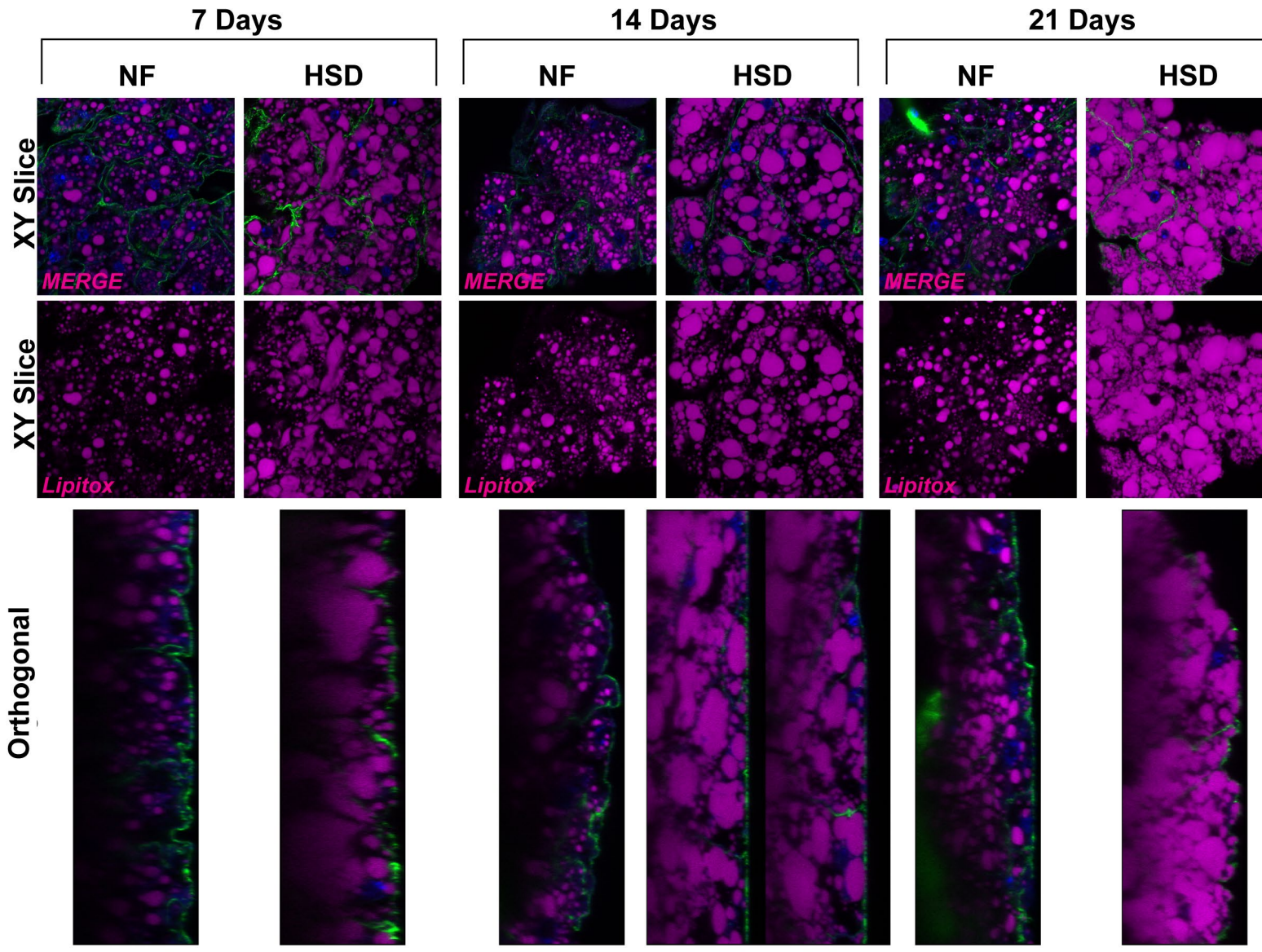


\section{Figure 2. DILP/FOXO accumulation and Lipid droplet morphology altered under HSD exposure.}

Slice of IPC neurons from W1118 adult fly brains fed either NF (A, top image) or HSD (A, bottom image) for 14 days, stained with Dilp5 antibody (green). Slices were taken from a confocal stack containing the entire IPC depth $(0.3 \mu \mathrm{m}$ increments). Analysis of fluorescent intensity shows Dilp5 is significantly lower in flies fed HSD (A, right). Bar graphs indicate average fluorescence for NF (white fill, green outline) or HSD (blue fill, red outline) with error bars indicating standard deviation. Slices of fly fat from day $14 \mathrm{NF}$ and HSD flies were taken and stained for FOXO (B). Slices were taken from a confocal stack of the fly fat, starting at the first sign of lamin marker, then following $0.3 \mu \mathrm{m}$ increments. Mean fluorescent intensity was measured in the nuclei of the fat and found FOXO to be more accumulated in the nuclei of flies fed HSD. Taken together, lower Dilp5 and high nuclear FOXO are indicative of insulin resistance in day 14 HSD W1118 flies. Representative slices of fly fat of NF and HSD fed flies at day 7 (2C, left), 14 (2C, middle), and 21 (2C, right) stained with lipidtox show larger and misshapen lipid droplets forming at all day under 21 day HSD. XY slices are shown at the top (2C) and orthogonal slices are shown at the bottom. 
3A. W1118 change in lipid class concentrations on HSD v. NF
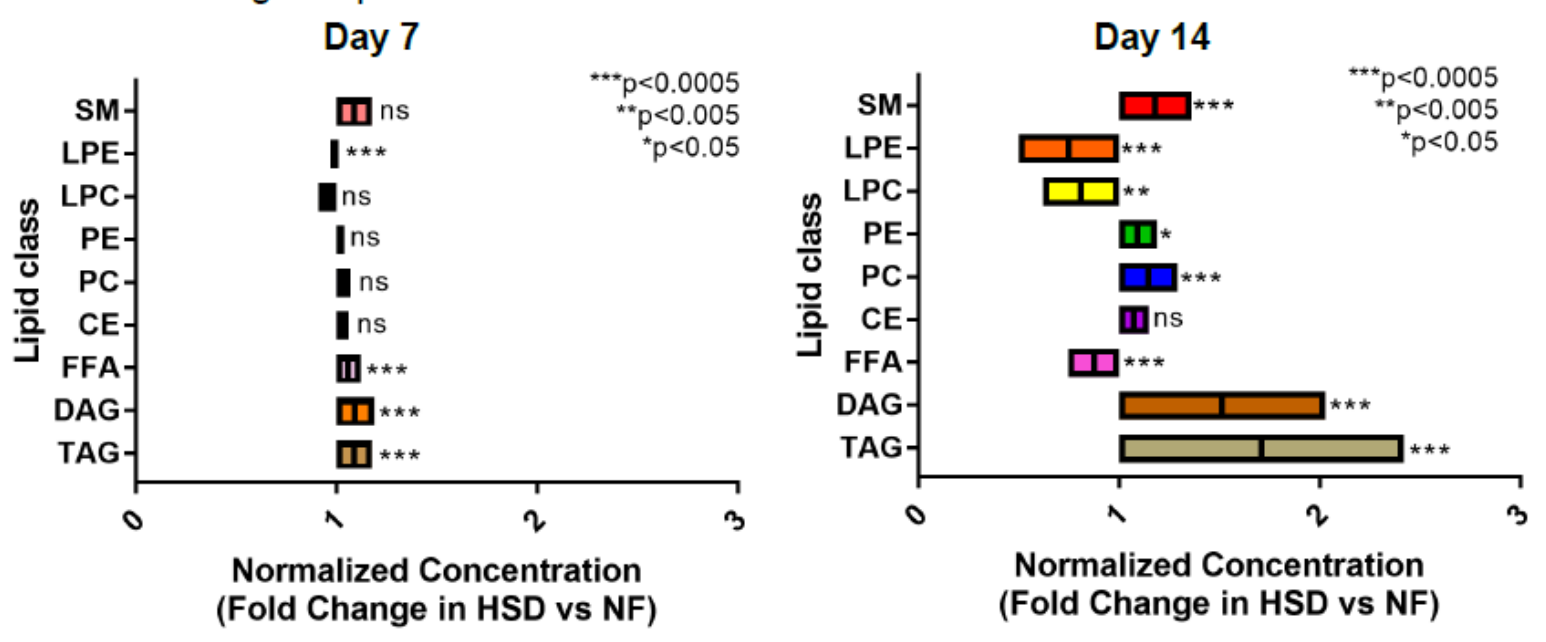

3B. W1118 PE and PC Concentration on different Diets
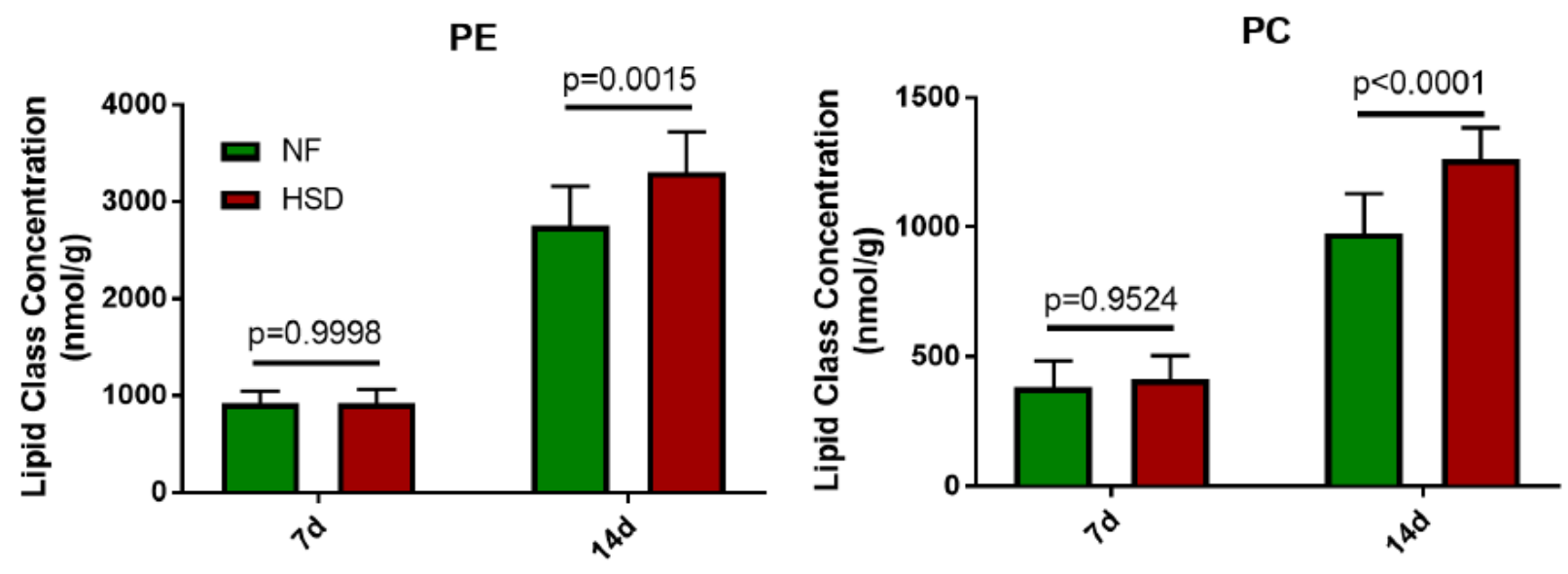


\section{Figure 3. Phospholipids are elevated in whole fly during extended HSD intake.}

Depicts the difference in concentration of all lipid classes in W1118 flies after 7 days (3A left panel) or 14 days (3A right panel) on HSD. Bars show the mean difference in fold change in the

5 HSD compared to NF, with data normalized to NF (Mean NF value equals 1). Flies on HSD show significantly higher Triacyl glyceride (TAG), Diacyl glyceride (DAG), and Free Fatty Acids (FFA) compared to NF flies as early as Day 7 where Hunger driven feeding is still maintained. However, by day 14 loss of hunger driven feeding also coincides with a large shift in the HSD lipidome, with a significant change not only to TAGs, DAGs, and FFAs, but increase in the phospholipids Phosphatidyl Ethanolamine (PE) and Phosphatidyl Choline (PC). The average concentrations of PE (3B, left panel) and PC (3B, right panel) in NF (green) and HSD (red) flies are directly compared to show the significant increase in concentration at Day 14. Lipidomics was performed using a targeted quantitative lipidyzer (Sciex 5500 Lipidyzer). 10 independent biological replicates were used for each diet and each day, with $n=10$ flies composing 1 biological replicate. Asterisks indicate significant changes with ${ }^{*} p$ value $<0.05$, ${ }^{* *} p$ value $<0.005$, and ${ }^{* *} p$ value $<0.0005$ 
bioRxiv preprint doi: https://doi.org/10.1101/2021.12.16.472854; this version posted December 21, 2021. The copyright holder for this preprint (which was not certified by peer review) is the author/funder, who has granted bioRxiv a license to display the preprint in perpetuity. It is made available under aCC-BY-NC-ND 4.0 International license.

4A. Difference in PECT Associated Phospholipid Classes (NF v. HSD)
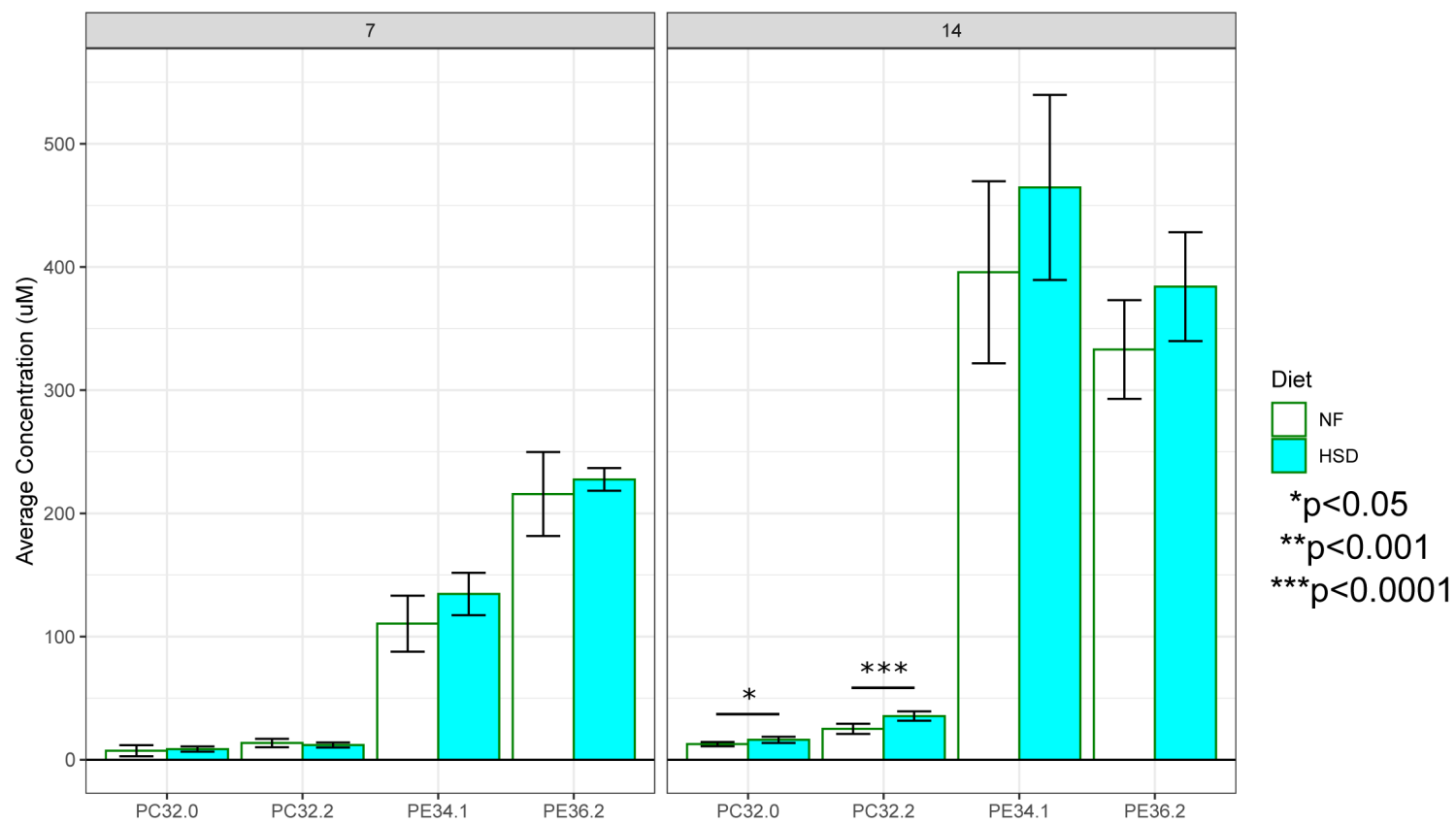

4B. Change in concentration of PE doublebond species in HSD v. NF

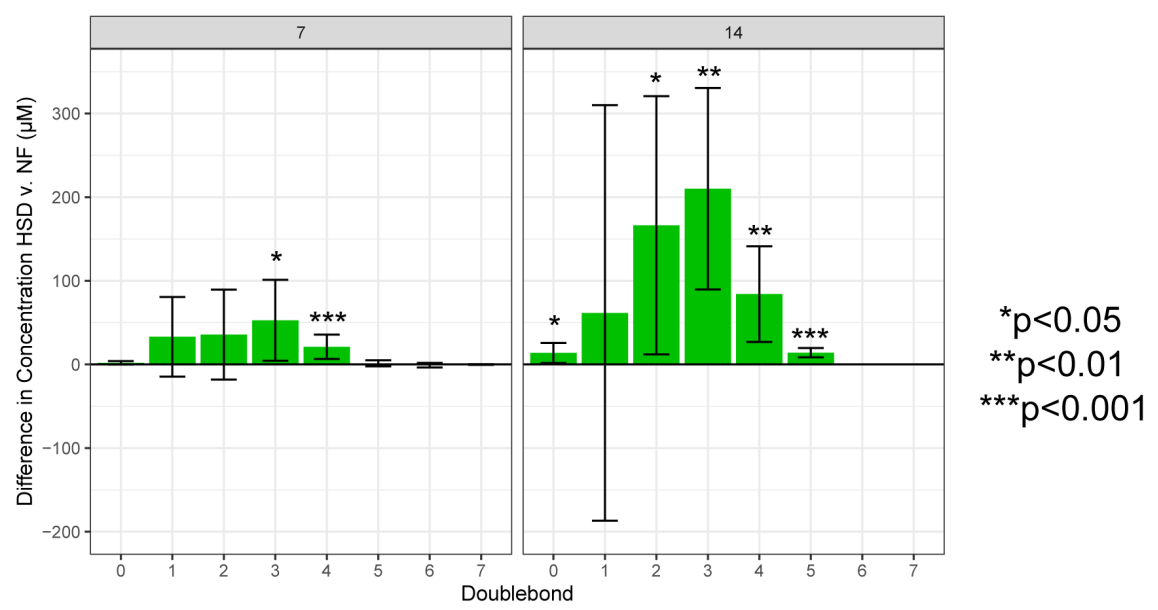

4C. Change in concentration of PC doublebond species in HSD v. NF

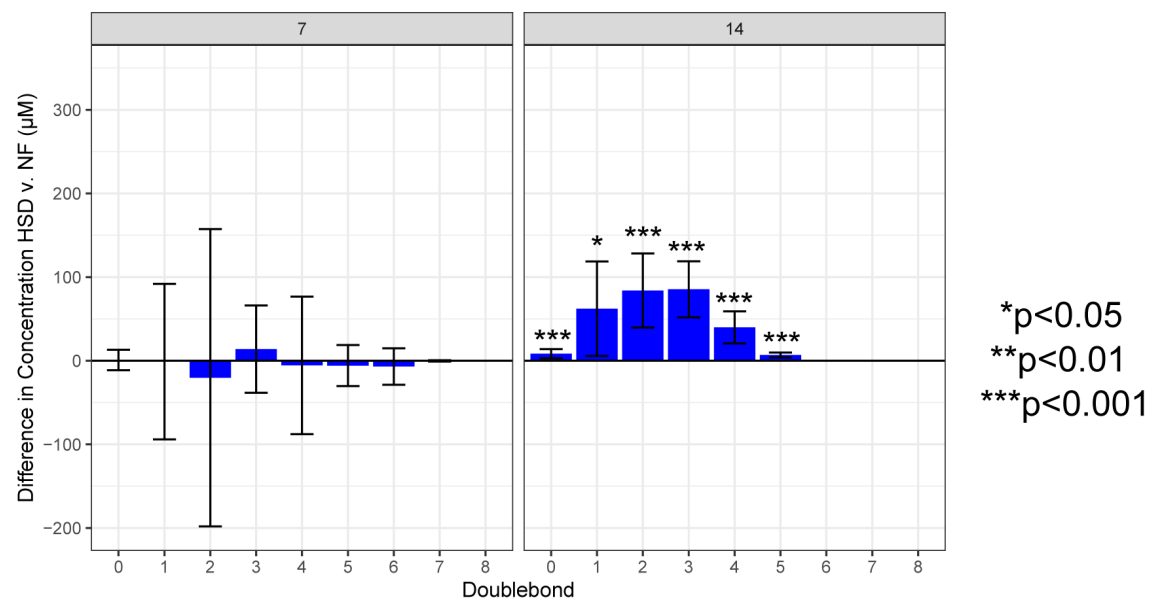




\section{Figure 4. Phospholipid species significantly altered in HSD are associated with PECT.}

Lipidomics data for PC and PE from Figure 3 was further assessed at the class level. In particular, it was found that PE and PC species and classes known to be associated with defects in PECT

5 from Clandinin et al. were significantly altered upon HSD treatment by day 14 (A). Individual classes are labelled on the x-axis. Bar graphs show the average concentration of each class for NF (white with green outline) and HSD (blue with green outline). Error bars indicate standard deviation $(n=10)$. Method for lipid quantification is the same as in Figure 3. Significant differences between NF and HSD are indicated with asterisks, with ${ }^{*} p$ value $<0.05$, ${ }^{* *} p$ value $<0.001$, and ${ }^{* \star *} p$ value $<0.0001$. Lipid species were assessed for changes in concentration based on doublebond number for PE (B) and PC (C). Concentrations for species with the same double bond number were summed together and the difference between concentrations of HSD to NF was compared for days 7 and 14. Error bars indicate standard deviation $(n=10)$. Significant differences between HSD and NF were calculated through two-way ANOVA with Holms Sidak correction. 
bioRxiv preprint doi: https://doi.org/10.1101/2021.12.16.472854; this version posted December 21, 2021. The copyright holder for this preprint (which was not certified by peer review) is the author/funder, who has granted bioRxiv a license to display the preprint in perpetuity. It is made available under aCC-BY-NC-ND 4.0 International license.

5A. Apoll accumulation in brain in PE Knockdown Flies (Fat Body Specific)
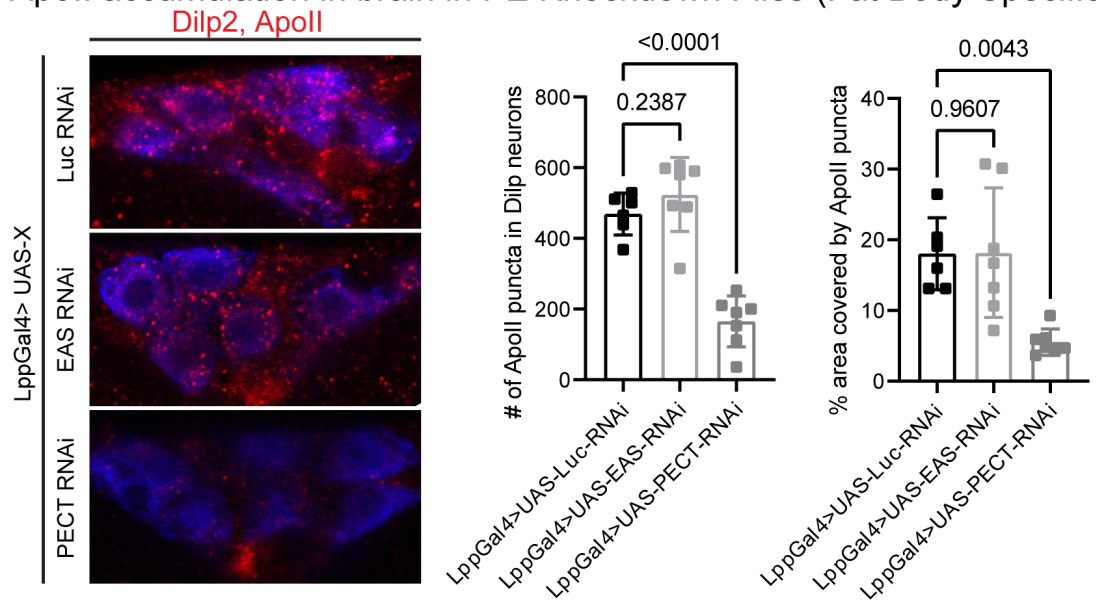

5B. Apoll accumulation in brain in PECT Overexpression Flies (Fat Body Specific)
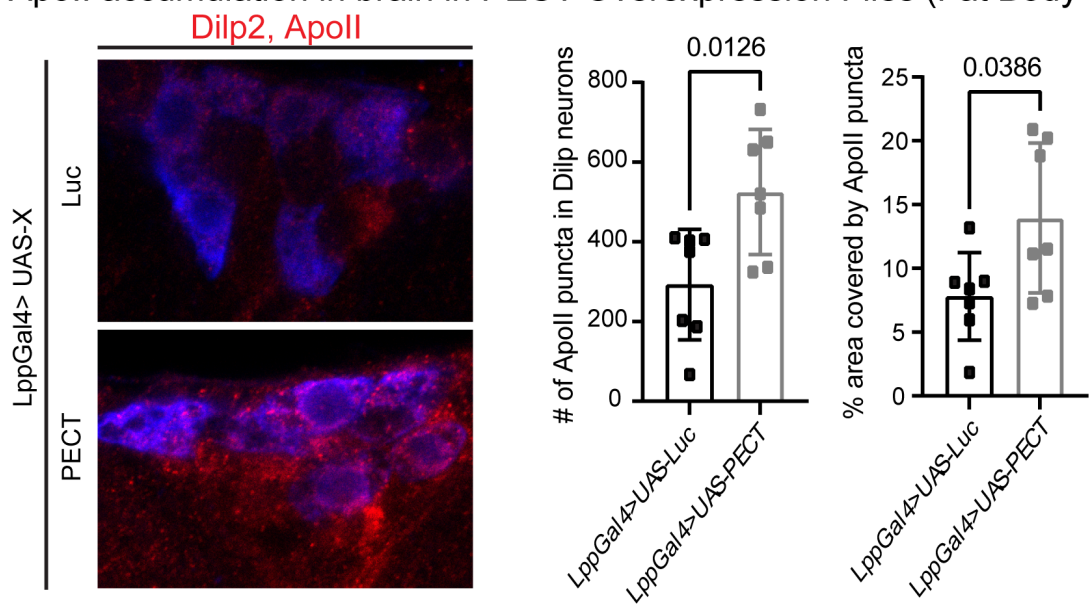


\section{Figure 5. Knockdown of PECT leads to decreased Apoll levels in the brain.}

Brain slices of day 14 flies stained for Apoll (red) and Dilp2 (blue) (A). Slices span the entire depth of the Dilp neuron at $0.3 \mu \mathrm{m}$ intervals. Representative slices are shown for Lpp-Gal4>UAS-Luc-

5 RNAi (A, top image), Lpp-Gal4>UAS-EAS-RNAi (A, middle image), and Lpp-Gal4>UAS-PECT-RNAi (A, bottom image). Number of Apoll puncta in the brain (5A, left bar graph) and area covered by said puncta (5A, right graph) were quantified through imageJ and determined that flies with a fat body specific knockdown of PECT show a significant decrease in number and coverage of Apoll in the brain. Bar graphs depict average values for 6-7 flies per genotype (depicted as squares) with errorbars indicating standard deviation. P-values were determined by one-way ANOVA with HolmSidak correction and are listed above. Conversely, a comparison of brain slices also stained with Apoll and Dilp2 in Lpp-Gal4>UAS-Luc (B, top image) and Lpp-Gal4>UAS-PECT (B, bottom image) were similarly quantified and found fat body specific overexpression led to more Apoll puncta number and coverage in the brain as determined by the unpaired t-test with Welch's correction. 
bioRxiv preprint doi: https://doi.org/10.1101/2021.12.16.472854; this version posted December 21, 2021. The copyright holder for this preprint (which was not certified by peer review) is the author/funder, who has granted bioRxiv a license to display the preprint in perpetuity. It is made available under aCC-BY-NC-ND 4.0 International license.

6A. FOXO accumulation in LG4>PECT-RNAi in fat
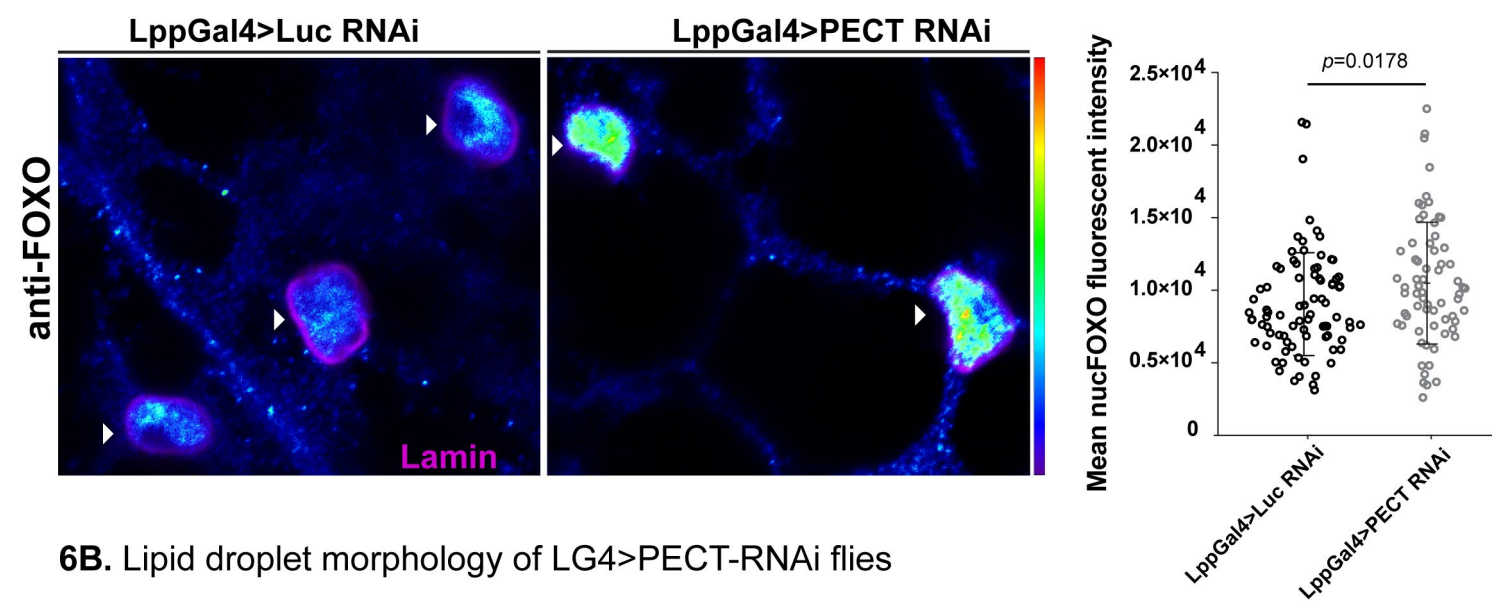

6B. Lipid droplet morphology of LG4>PECT-RNAi flies

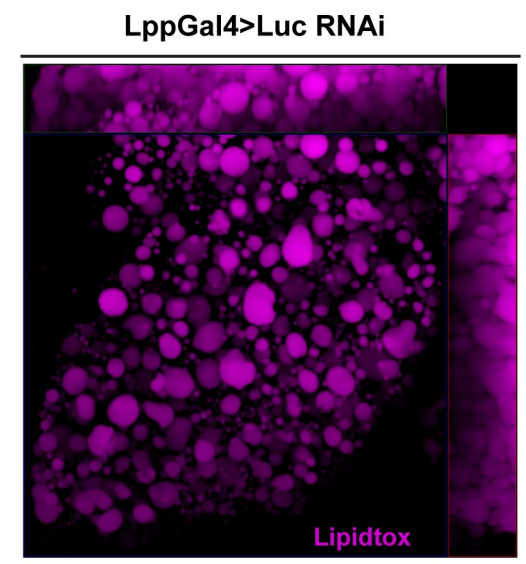

LppGal4>PECT RNAi

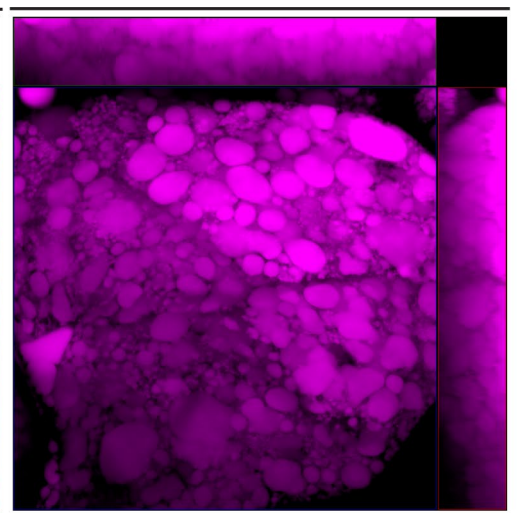




\section{Figure 6. PECT knockdown in fly fat alters FOXO nuclear accumulation and lipid droplet morphology.}

Confocal single plane images of Lpp-Gal4>UAS-Luc-RNAi (6A, left) and Lpp-Gal4>UAS-PECT-RNAi

5 (6A, middle) fly fat stained for anti-FOXO (blue to green, intensity based) and lamin (pink) on day

14. Confocal stack of the fat body nuclei was taken at the first plane with lamin detected, then measured at $0.3 \mu \mathrm{m}$ intervals. Nuclear accumulation of FOXO is significantly higher in LppGal4>UAS-PECT-RNAi, which is seen as a marker for insulin resistance (6A, right) ${ }^{54,55}$. Error bars indicate standard deviation. Lipid morphology was observed in representative Lpp-Gal4>UASLuc-RNAi (6B, left) and Lpp-Gal4>UAS-PECT-RNAi (6B, right) slices by staining with lipidtox. XY slices are the center images and the edges are the orthogonal slices. Flies with PECT knockdown larger and more clustered lipid droplets than their control counterparts. 
bioRxiv preprint doi: https://doi.org/10.1101/2021.12 16.472854; this version posted December 21, 2021. The copyright holder for this preprint (which was not certified by peer review) is the author/funder, who has granted bioRxiv a license to display the preprint in perpetuity. It is made available under aCC-BY-NC-ND 4.0 International license.

7A. Day 14 feeding behavior in PE/PC biosynthesis knockdown flies (fat body specific)
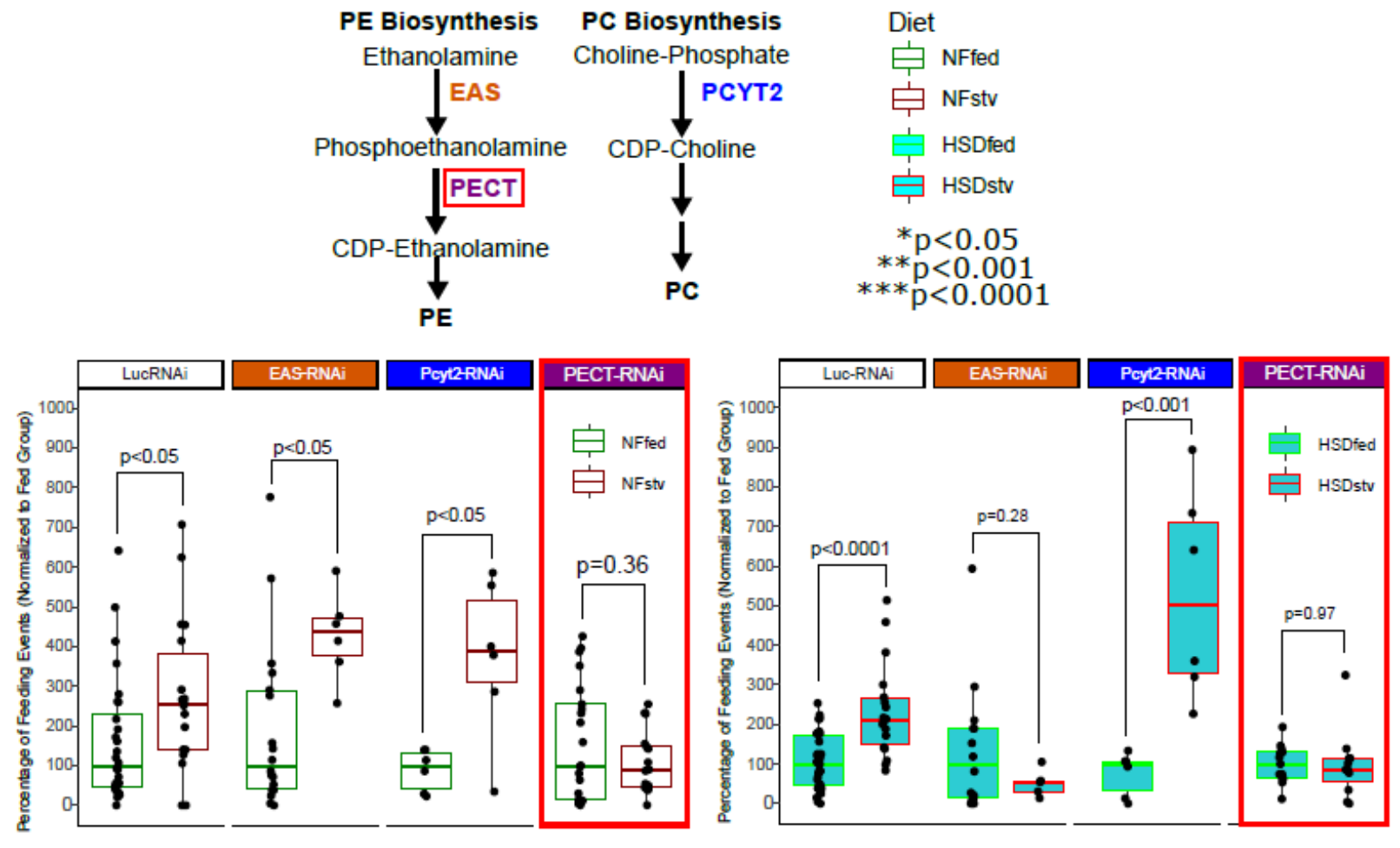

7B. PECT Overexpression Hunger Driven Feeding Behavior (NF and HSD)
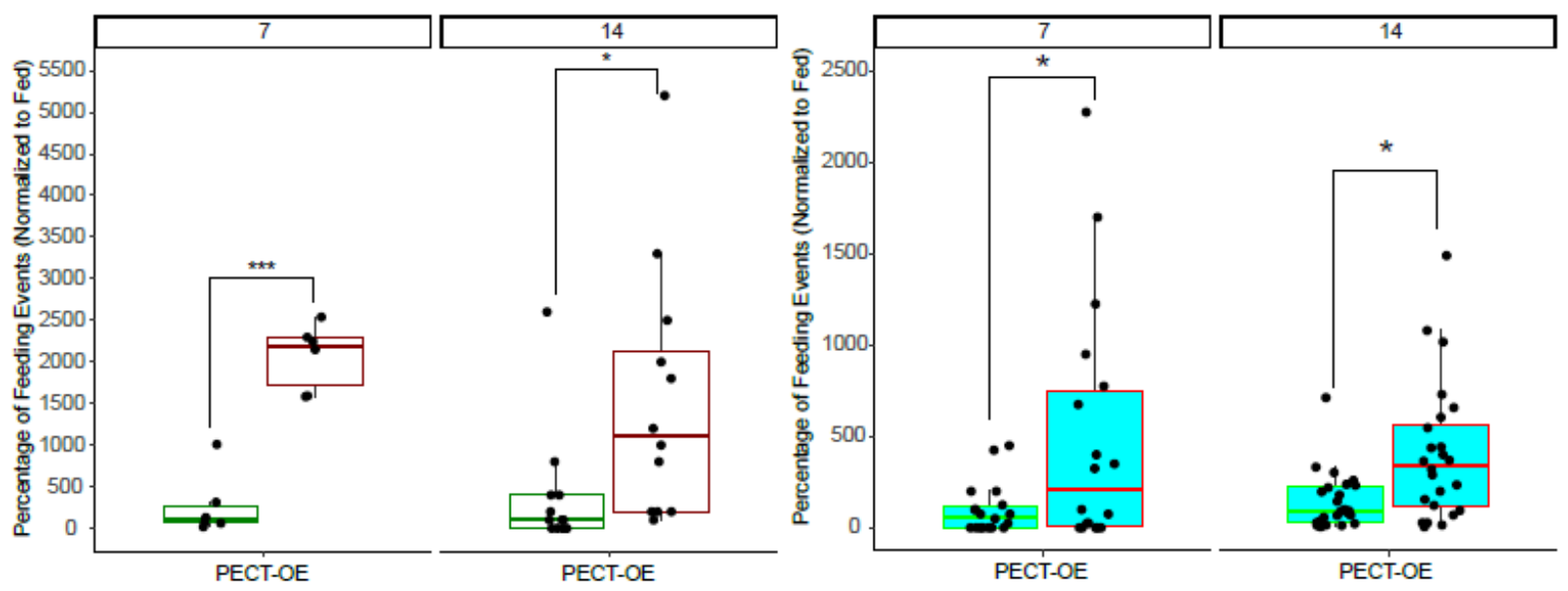
Figure 7. Hunger Driven Feeding behavior altered through fat body specific Phospholipid biosynthesis.

With evidence of PC and PE being influenced by HSD, we generated mutant fly lines to manipulate $\mathrm{PC} / \mathrm{PE}$ biosynthesis in the fat body to determine if manipulations alter HDF. Figure 7A shows the HDF responses of Lpp-Gal4>UAS-EAS-RNAi (orange), Lpp-Gal4>UAS-PCYT2-RNAi (blue), and LppGal4>PECT-RNAi (purple) after 14 days of NF (7A, middle panel) or HSD (7A, right panel) following a 7 day incubation on NF media. Pathway for PC and PE biosynthesis with relevant enzymes is shown in 7a (left panel). A knockdown of PECT in the fat resulted in HDF loss for both NF and HSD flies and a knockdown of EAS had a loss of HDF for the HSD flies. Impacting the PC biosynthesis pathway through PCYT2 showed no significant effect on HDF. By using Lpp-Gal4>UAS-PECT overexpression line (PECT-OE, Figure 7B), we see a partial rescue of feeding behavior in both NF and HSD treated flies. Black dots represent and individual flies feeding. 16 hours prior to feeding measurements, flies were flipped either to fresh media (Fed group, green outline) or moved to a 1\% sucrose/agar media (Starved (Stv) group, red outline). Feeding events were automatically recorded for individual flies using a Fly Liquid Interaction Counter (FLIC). Total feeding events for the first three hours in the FLIC were tallied for each fly and the respective fed and starved groups were normalized as a percentage of feeding events, with the median fly feeding events for the fed group being considered as $100 \%$ feeding. Both fed and starved counterparts were measured in parallel on the same day. Asterisks indicate significance with ${ }^{*} p$ value $<0.01$, ${ }^{\star *} p$ value $<0.001$, and *** $p$ value $<0.0001$ 
bioRxiv preprint doi: https://doi.org/10.1101/2021.12 16.472854 this version posted December 21, 2021. The copyright holder for this preprint (which was not certified by peer review) is the author/funder, who has granted bioRxiv a license to display the preprint in perpetuity. It is made available under aCC-BY-NC-ND 4.0 International license.

S1A. W1118 Baseline Feeding on NF and HSD over time
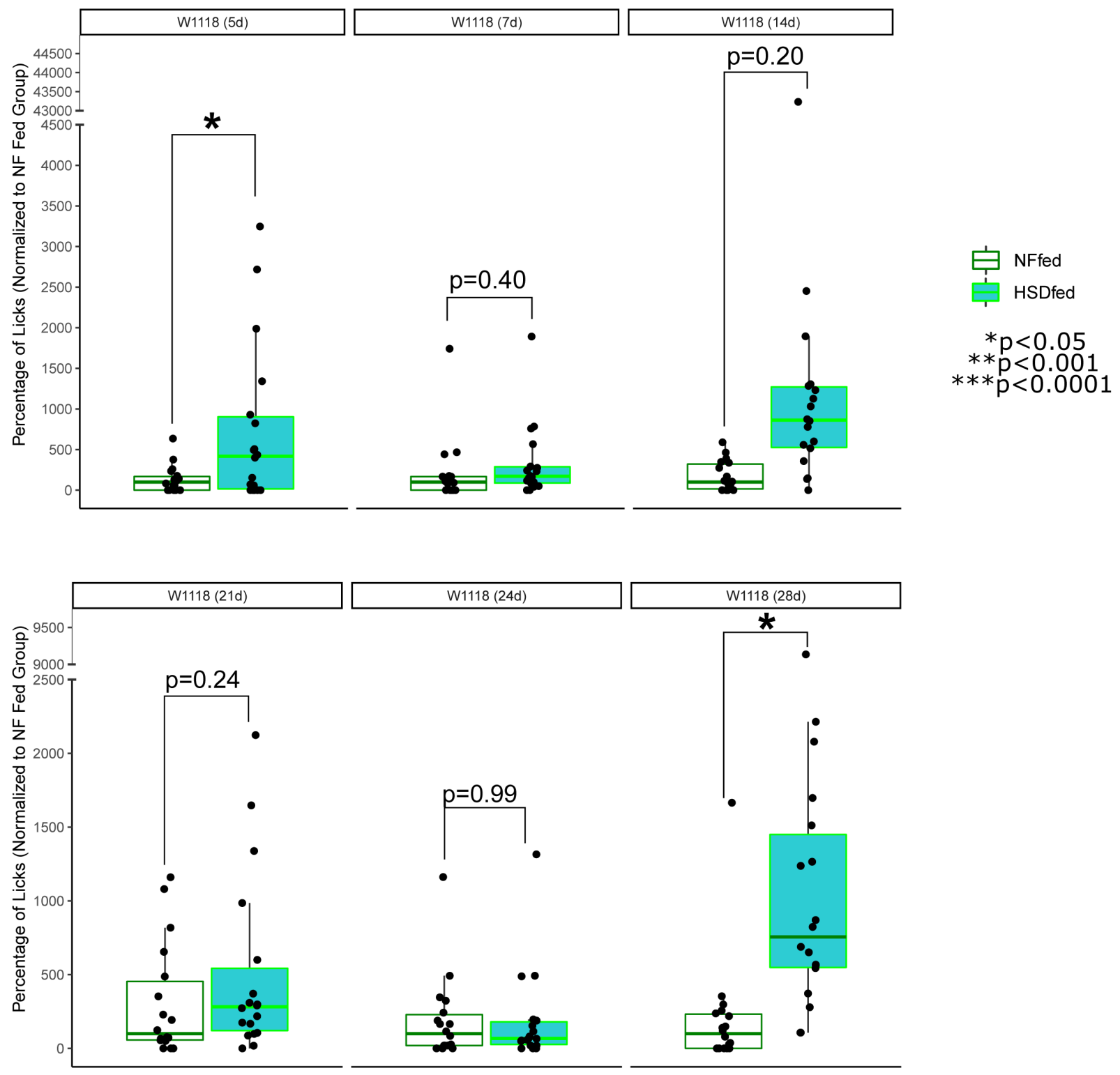


\section{Figure S1. Baseline levels of feeding in W1118 elevated over time on HSD.}

HSD flies have higher baseline feeding compared to NF throughout adult lifespan. Black dots represent and individual flies feeding. Feeding events were automatically recorded for individual

5 flies using a Fly Liquid Interaction Counter (FLIC). Total feeding events for the first three hours in the FLIC were tallied for each fly and the respective NF fed and HSD fed groups were normalized as a percentage of feeding events, with the median fly feeding events for the NF fed group being considered as $100 \%$ feeding. Both NF and HSD fed counterparts were measured in parallel on the same day. Asterisks indicate significance with ${ }^{*} p$ value $<0.01$, ${ }^{* *} p$ value $<0.001$, and ${ }^{* \star *} p$ value $<0.0001$. 
bioRxiv preprint doi: https://doi.org/10.1101/2021.12.16.472854; this version posted December 21,2021 . The copyright holder for this preprint (which was not certified by peer review) is the author/funder, who has granted bioRxiv a license to display the preprint in perpetuity. It is made available under aCC-BY-NC-ND 4.0 International license.

S2A. Validation of Apoll antibody

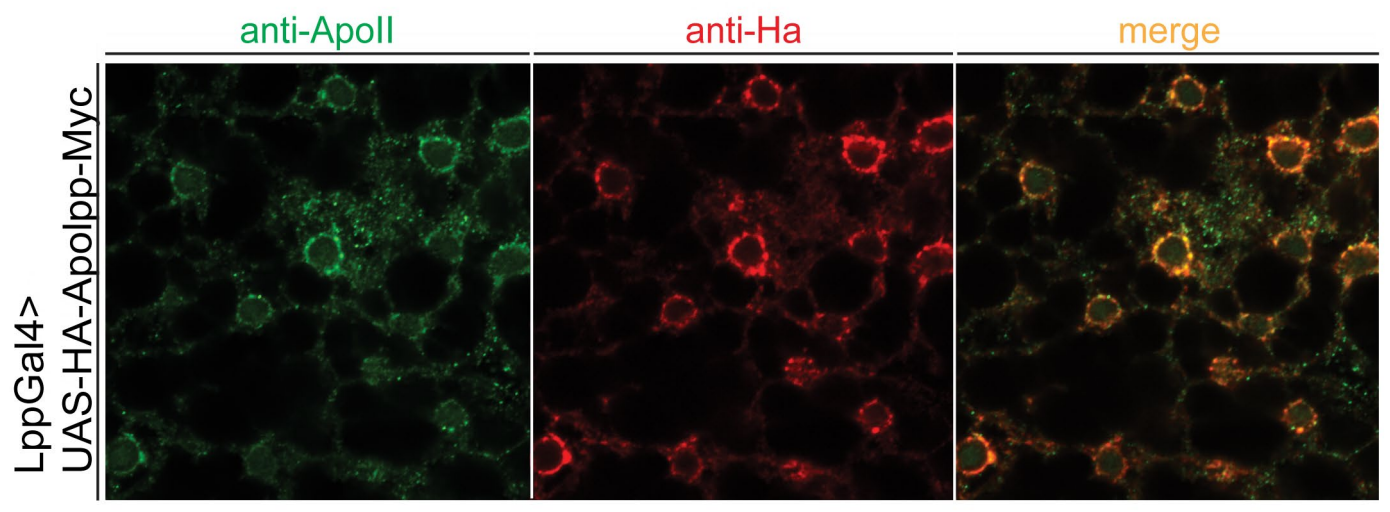

S2B. FOXO accumulation in W1118 (6 hours after diet treatment)

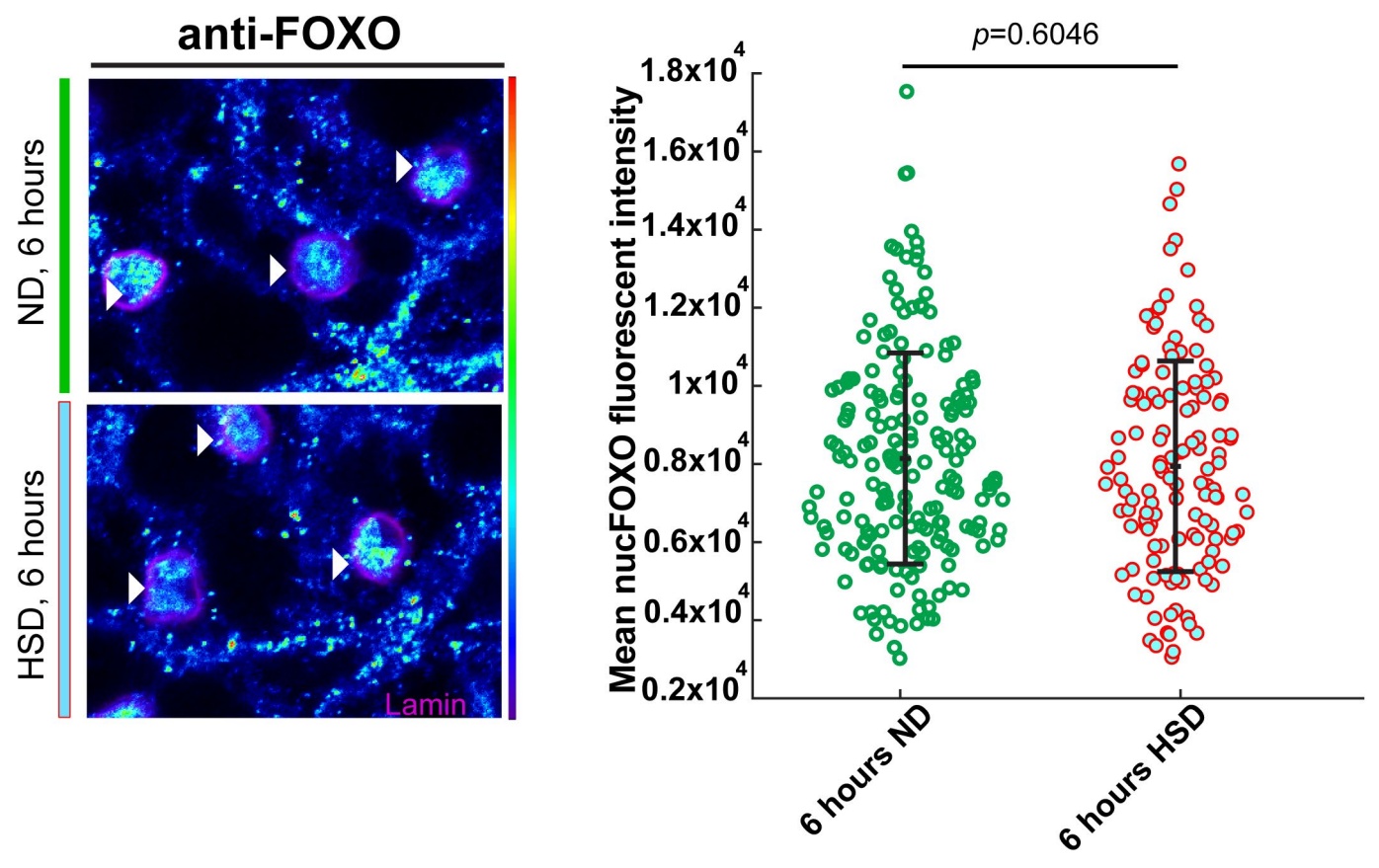


Figure S2. Validation of Apoll antibody and lack of FOXO accumulation after 6 hours of HSD. S2A) Depicts confocal single plane image of LG4>HA-Apolpp-myc fly fat body stained for Apoll antibody (green), HA antibody (red), and a merge of the two images. Merge indicates HA and Apoll stain are occurring in the same regions. S2B) Confocal single plane images of W1118 flies given normal diet (S4B, top) or HSD (S4B, bottom). fly fat stained for anti-FOXO (blue to green, intensity based) and lamin (pink) for six hours after initial 7 day incubation after eclosion. Confocal stack of the fat body nuclei was taken at the first plane with lamin detected, then measured at $0.3 \mu \mathrm{m}$ intervals. Nuclear accumulation of FOXO is unchanged in W1118 flies after an acute six hour period on HSD. Error bars indicate standard deviation. 
bioRxiv preprint doi: $\mathrm{https}$ //doi.org/10.1101/2021.12 16.472854; this version posted December 21, 2021. The copyright holder for this preprint (which was not certified by peer review) is the author/funder, who has granted bioRxiv a license to display the preprint in perpetuity. It is made available under aCC-BY-NC-ND 4.0 International license.

S3A. PISD-RNAi Hunger Driven Feeding Behavior
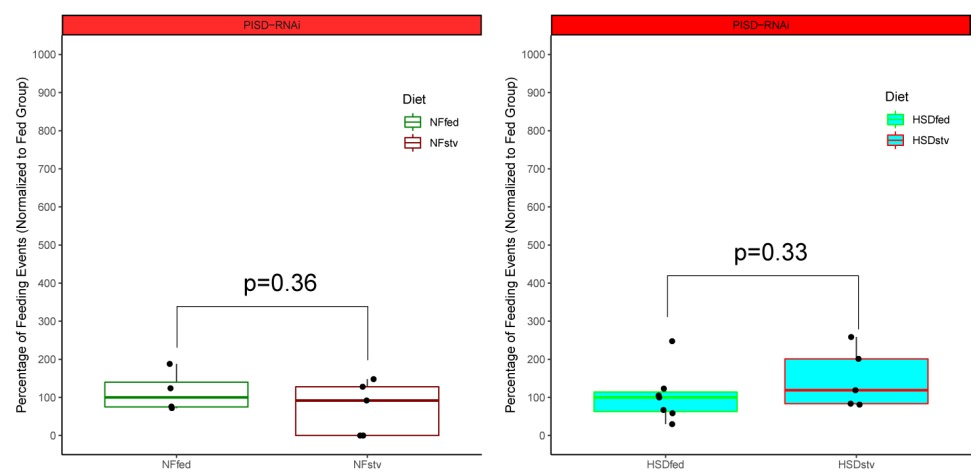

S3B. PECT Expression in Mutant Lines
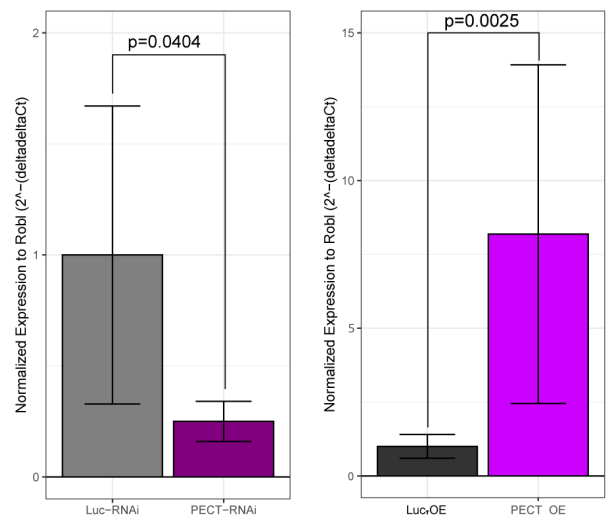

S3C. PECT Expression in W1118 over time

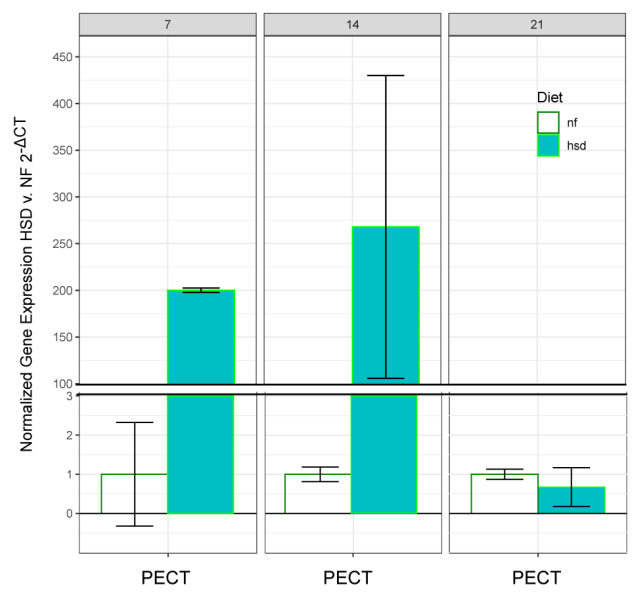




\section{Figure S3. PISD knockdown also disrupts Hunger driven feeding and PECT expression show} large increase on HSD. With the knowledge that PECT knockdown was able to impact HDF, we measured HDF in Lpp-Gal4>UAS-PISD-RNAi flies which is the equivalent PE biosynthesis pathway

5 in the mitochondria. Indeed, we also see a similar loss of HDF feeding on day 14 as in the PECT knockdown (Figure S3A). Expression of PECT was confirmed to be downregulated in the LG4>PECT-RNAi flies (S3B left panel) and upregulated in the LppGal4>UAS-PECT-overexpression (S3B right panel). qPCR was performed on day 14 from RNA isolated from fly fat bodies. 15 fat bodies were used for each biological replicate and three independent biological replicates were used. Finally, PECT expression was also measured by qPCR in W1118 flies in both NF (white with green outline) and HSD (blue with green outline) on days 7, 14, and 21 (S3C). 15 whole flies were used for each biological replicate and three independent biological replicates were used. For all qPCR graphs, gene expression is normalized to the control gene expression (Robl). Expression was then normalized to NF, with average PECT of normal fed being 1. Error bars indicate standard deviation. 
bioRxiv preprint doi: https://doi.org/10.1101/2021.12.16.472854; this version posted December 21, 2021. The copyright holder for this preprint (which was not certified by peer review) is the author/funder, who has granted bioRxiv a license to display the preprint in perpetuity. It is made available under aCC-BY-NC-ND 4.0 International license.

S4A. PE composition by class on NF (day 7)

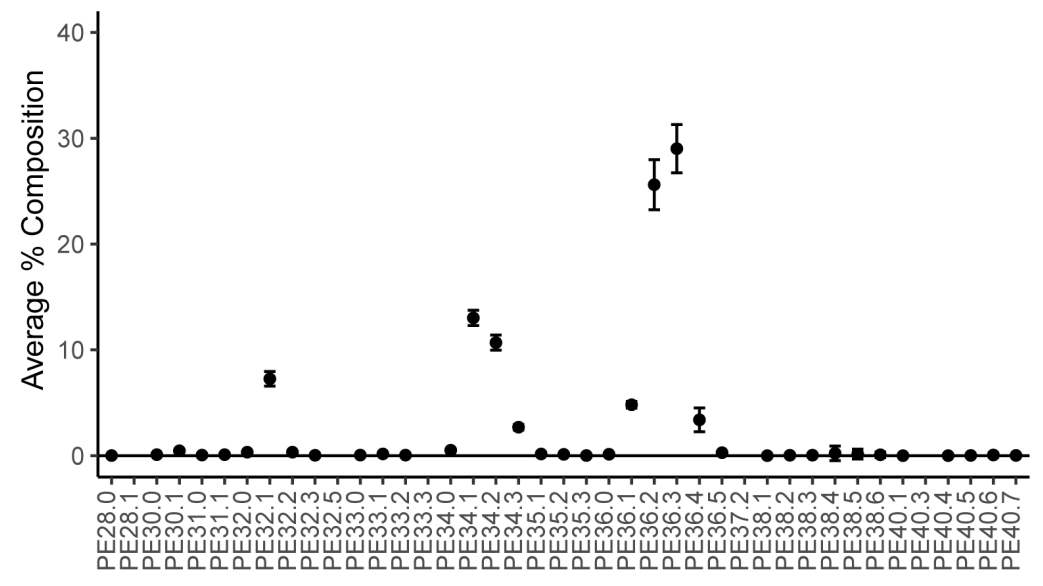

S4B. PC composition by class on NF (day 7)

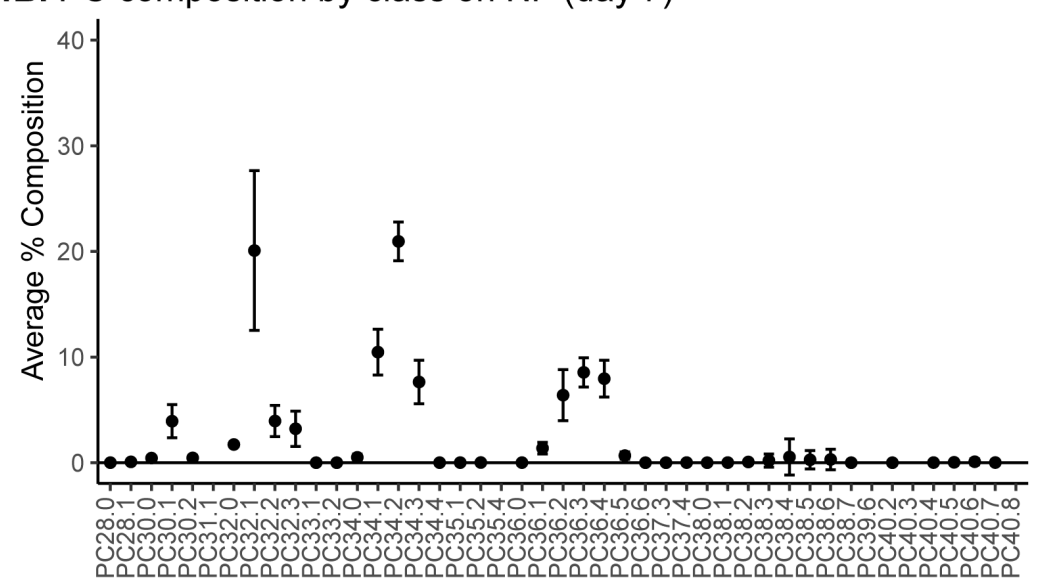

S4C. Difference in PECT Associated Phospholipid Classes (NF v. HSD)
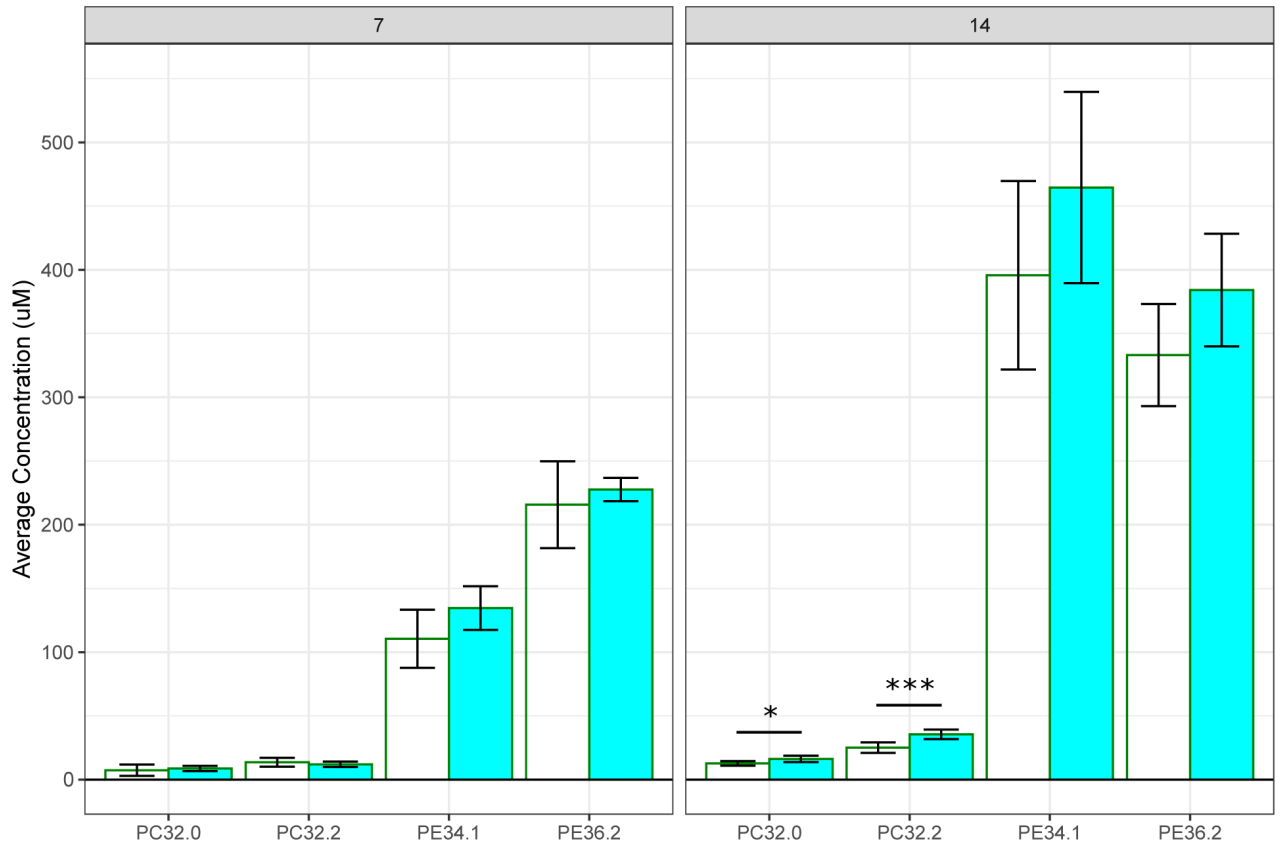

Diet
$\square{ }^{\mathrm{NF}}$
$\square{ }_{\text {HSD }}^{*} p<0.05$
${ }^{* *} p<0.001$
${ }^{* * *} \mathrm{p}<0.0001$ 
Figure S4. W1118 Phospholipid concentration significantly increased on HSD by day 14 for PECT associated Classes. S4A) graph showing \% lipid composition for all PE and PC (S4B) classes in day 7 NF flies. Composition was averaged amongst 10 biological replicates ( $n=10$ 5 flies/replicate). Error bars indicate standard deviation. S3C measures the concentration of both NF and HSD fed flies for PC and PE classes associated with PECT based on Clandinin et al. for day 7 (left graph) and day 14 (right graph). Upon 14 days of HSD, flies show higher concentration of all classes with significant increases for PC32.0 and PC32.2 compared to NF group. Bars plot average concentration amongst 10 biological replicates ( $n=10$ flies/replicate) and error bars 10 indicate standard deviation. Significance was calculated by t-test and identified by * $p$ value $<0.05$, ${ }^{\star \star} p$ value $<0.001$, and ${ }^{\star \star *} p$ value $<0.0001$ 


\section{Acknowledgements}

We would like to thank Dr. Pierre Leopold for generously donating the FOXO antibody used in this manuscript. We are grateful to Thomas Clandinin for gifting the UAS-PECT 5 transgenic flies. We would also like to thank the team at the Northwest Metabolomics Research Center for their support in lipidomic profiling. This work was possible due to grants awarded to AR from NIGMS (GM124593), and New Development funds from Fred Hutch Cancer Research Center. KPK was supported by the NIH Chromosome Metabolism and Cancer Training Grant (T32CA009657) and is currently supported as an NSF Post Doctoral Research Fellow (NSF Award \#2109398). Genomic reagents from the DGRC which is funded by NIH grant 2P40D010949 were used in this study. Stocks obtained from the Bloomington Drosophila Stock Center (NIH P40OD018537) and Transgenic RNAi Resource Project (NIGMS R01 GM084947 and NIGMS P41 GM132087) were used in this study.

\section{Author Contributions}

Conceptualization: AR

Investigation: MEP, CES, AEB, KPK, MAA, ZHG

Formal analysis/Data Curation: AEB, KPK, ZHG, CES, MAA

Visualization: KPK, MAA, JD

20 Supervision: AR

Writing- original draft: KPK

Writing- review and editing: $A R, M A A, K P K$

Funding Acquisition: AR

\section{Competing Interests}

The authors declare no competing interests. 


\section{Citations}

1. Wang, J., Obici, S., Morgan, K., Barzilai, N., Feng, Z., and Rossetti, L. (2001). Overfeeding Rapidly Induces Leptin and Insulin Resistance. Diabetes 50, 2786 LP - 2791.

2. Adeva-Andany, M.M., González-Lucán, M., Fernández-Fernández, C., Carneiro-Freire, N., Seco-Filgueira, M., and Pedre-Piñeiro, A.M. (2019). Effect of diet composition on insulin sensitivity in humans. Clin. Nutr. ESPEN 33, 29-38.

3. Vasselli, J.R., Scarpace, P.J., Harris, R.B.S., and Banks, W.A. (2013). Dietary components in the development of leptin resistance. Adv. Nutr. 4, 164-175.

4. Makimura, H., Mizuno, T.M., Isoda, F., Beasley, J., Silverstein, J.H., and Mobbs, C. V (2003).

Role of glucocorticoids in mediating effects of fasting and diabetes on hypothalamic gene expression. BMC Physiol. 3, 5.

5. Ahima, R.S., Prabakaran, D., Mantzoros, C., Qu, D., Lowell, B., Maratos-Flier, E., and Flier, J.S. (1996). Role of leptin in the neuroendocrine response to fasting. Nature 382, 250-252.

6. Veniant, M.M., and LeBel, C.P. (2003). Leptin: from animals to humans. Curr. Pharm. Des. 9, 811-818.

7. Leopold, P., and Perrimon, N. (2007). Drosophila and the genetics of the internal milieu. Nature 450, 186-188.

8. Delanoue, R., Meschi, E., Agrawal, N., Mauri, A., Tsatskis, Y., McNeill, H., and Léopold, P. (2016). Drosophila insulin release is triggered by adipose Stunted ligand to brain Methuselah receptor. Science 353, 1553-1556.

9. Meschi, E., Léopold, P., and Delanoue, R. (2019). An EGF-Responsive Neural Circuit Couples Insulin Secretion with Nutrition in Drosophila. Dev. Cell 48, 76-86.e5.

10. Smith, W.W., Thomas, J., Liu, J., Li, T., and Moran, T.H. (2014). From fat fruit fly to human obesity. Physiol. Behav. 136, 15-21.

11. Arrese, E.L., and Soulages, J.L. (2010). Insect fat body: energy, metabolism, and regulation. Annu. Rev. Entomol. 55, 207-225.

12. Gáliková, M., and Klepsatel, P. (2018). Obesity and Aging in the Drosophila Model. Int. J. 
Mol. Sci. 19, 1896.

13. Palanker Musselman, L., Fink, J.L., Narzinski, K., Ramachandran, P.V., Sukumar Hathiramani, S., Cagan, R.L., and Baranski, T.J. (2011). A high-sugar diet produces obesity and insulin resistance in wild-type Drosophila. Dis. Model. Mech. 4, 842-849.

5 14. Walker, S.J., Goldschmidt, D., and Ribeiro, C. (2017). Craving for the future: the brain as a nutritional prediction system. Curr. Opin. Insect Sci. 23, 96-103.

15. Kim, S.K., Tsao, D.D., Suh, G.S.B., and Miguel-Aliaga, I. (2021). Discovering signaling mechanisms governing metabolism and metabolic diseases with Drosophila. Cell Metab. 33, 1279-1292.

16. Tennessen, J.M., Barry, W.E., Cox, J., and Thummel, C.S. (2014). Methods for studying metabolism in Drosophila. Methods 68, 105-115.

17. May, C.E., Vaziri, A., Lin, Y.Q., Grushko, O., Khabiri, M., Wang, Q.-P., Holme, K.J., Pletcher, S.D., Freddolino, P.L., Neely, G.G., et al. (2019). High Dietary Sugar Reshapes Sweet Taste to Promote Feeding Behavior in Drosophila melanogaster. Cell Rep. 27, 1675-1685.e7.

18. Small, D.M. (2009). Individual differences in the neurophysiology of reward and the obesity epidemic. Int. J. Obes. (Lond). 33 Suppl 2, S44-S48.

19. Volkow, N.D., Wang, G.-J., and Baler, R.D. (2011). Reward, dopamine and the control of food intake: implications for obesity. Trends Cogn. Sci. 15, 37-46.

20. Musso, P.-Y., Junca, P., Jelen, M., Feldman-Kiss, D., Zhang, H., Chan, R.C.W., and Gordon, M.D. (2019). Closed-loop optogenetic activation of peripheral or central neurons modulates feeding in freely moving Drosophila. Elife 8, e45636.

21. Pierre-Yves, M., Pierre, J., and D., G.M. (2021). A neural circuit linking two sugar sensors regulates satiety-dependent fructose drive in Drosophila. Sci. Adv. 7, eabj0186.

22. Stanley, M., Ghosh, B., Weiss, Z.F., Christiaanse, J., and Gordon, M.D. (2021). Mechanisms of lactic acid gustatory attraction in Drosophila. Curr. Biol. 31, 3525-3537.e6.

23. Ro, J., Harvanek, Z.M., and Pletcher, S.D. (2014). FLIC: High-Throughput, Continuous Analysis of Feeding Behaviors in Drosophila. PLoS One 9, e101107.

24. Pendergast, J.S., Wendroth, R.H., Stenner, R.C., Keil, C.D., and Yamazaki, S. (2017). 
mPeriod2 (Brdm1) and other single Period mutant mice have normal food anticipatory activity. Sci. Rep. 7, 15510.

25. Pendergast, J.S., Branecky, K.L., Huang, R., Niswender, K.D., and Yamazaki, S. (2014). Wheel-running activity modulates circadian organization and the daily rhythm of eating behavior. Front. Psychol. 5, 177.

26. Deshpande, S.A., Carvalho, G.B., Amador, A., Phillips, A.M., Hoxha, S., Lizotte, K.J., and Ja, W.W. (2014). Quantifying Drosophila food intake: comparative analysis of current methodology. Nat. Methods 11, 535-540.

27. Post, S., Liao, S., Yamamoto, R., Veenstra, J.A., Nässel, D.R., and Tatar, M. (2019). Drosophila insulin-like peptide dilp1 increases lifespan and glucagon-like Akh expression epistatic to dilp2. Aging Cell 18, e12863.

28. Shinoda, W. (2016). Permeability across lipid membranes. Biochim. Biophys. Acta Biomembr. 1858, 2254-2265.

29. Ben M’barek, K., Ajjaji, D., Chorlay, A., Vanni, S., Forêt, L., and Thiam, A.R. (2017). ER Membrane Phospholipids and Surface Tension Control Cellular Lipid Droplet Formation. Dev. Cell 41, 591-604.e7.

30. Choudhary, V., Golani, G., Joshi, A.S., Cottier, S., Schneiter, R., Prinz, W.A., and Kozlov, M.M. (2018). Architecture of Lipid Droplets in Endoplasmic Reticulum Is Determined by Phospholipid Intrinsic Curvature. Curr. Biol. 28, 915-926.e9.

31. Sharma, N.K., Langberg, K.A., Mondal, A.K., and Das, S.K. (2013). Phospholipid Biosynthesis Genes and Susceptibility to Obesity: Analysis of Expression and Polymorphisms. PLoS One 8, e65303.

32. Chang, W., Hatch, G.M., Wang, Y., Yu, F., and Wang, M. (2019). The relationship between phospholipids and insulin resistance: From clinical to experimental studies. J. Cell. Mol. Med. 23, 702-710.

33. Anjos, S., Feiteira, E., Cerveira, F., Melo, T., Reboredo, A., Colombo, S., Dantas, R., Costa, E., Moreira, A., Santos, S., et al. (2019). Lipidomics Reveals Similar Changes in Serum Phospholipid Signatures of Overweight and Obese Pediatric Subjects. J. Proteome Res. 18, 3174-3183. 
34. Lim, H.-Y., Wang, W., Wessells, R.J., Ocorr, K., and Bodmer, R. (2011). Phospholipid homeostasis regulates lipid metabolism and cardiac function through SREBP signaling in Drosophila. Genes Dev. 25, 189-200.

35. Tsai, J.W., Kostyleva, R., Chen, P.-L., Rivas-Serna, I.M., Clandinin, M.T., Meinertzhagen, I.A., and Clandinin, T.R. (2019). Transcriptional Feedback Links Lipid Synthesis to Synaptic Vesicle Pools in Drosophila Photoreceptors. Neuron 101, 721-737.e4.

36. van Dam, E., van Leeuwen, L.A.G., Dos Santos, E., James, J., Best, L., Lennicke, C., Vincent, A.J., Marinos, G., Foley, A., Buricova, M., et al. (2020). Sugar-Induced Obesity and Insulin Resistance Are Uncoupled from Shortened Survival in Drosophila. Cell Metab. 31, 710725.e7.

37. Suzuki, K., Jayasena, C.N., and Bloom, S.R. (2012). Obesity and appetite control. Exp. Diabetes Res. 2012, 824305.

38. Ellacott, K.L.J., Morton, G.J., Woods, S.C., Tso, P., and Schwartz, M.W. (2010). Assessment of feeding behavior in laboratory mice. Cell Metab. 12, 10-17.

39. McGrath, T.M., Spreckley, E., Rodriguez, A.F., Viscomi, C., Alamshah, A., Akalestou, E., Murphy, K.G., and Jones, N.S. (2019). The homeostatic dynamics of feeding behaviour identify novel mechanisms of anorectic agents. PLoS Biol. 17, e3000482-e3000482.

40. Lee, S., and Dong, H.H. (2017). FoxO integration of insulin signaling with glucose and lipid metabolism. J. Endocrinol. 233, R67-R79.

20 41. Gross, D.N., van den Heuvel, A.P.J., and Birnbaum, M.J. (2008). The role of FoxO in the regulation of metabolism. Oncogene $27,2320-2336$.

42. Kim, J.I., Huh, J.Y., Sohn, J.H., Choe, S.S., Lee, Y.S., Lim, C.Y., Jo, A., Park, S.B., Han, W., and Kim, J.B. (2015). Lipid-overloaded enlarged adipocytes provoke insulin resistance independent of inflammation. Mol. Cell. Biol. 35, 1686-1699.

43. Ammar, M.R., Kassas, N., Chasserot-Golaz, S., Bader, M.-F., and Vitale, N. (2013). Lipids in Regulated Exocytosis: What are They Doing? Front. Endocrinol. (Lausanne). 4, 125.

44. Sunshine, H., and Iruela-Arispe, M.L. (2017). Membrane lipids and cell signaling. Curr. Opin. Lipidol. 28, 408-413. 
45. Palm, W., Sampaio, J.L., Brankatschk, M., Carvalho, M., Mahmoud, A., Shevchenko, A., and Eaton, S. (2012). Lipoproteins in Drosophila melanogaster-Assembly, Function, and Influence on Tissue Lipid Composition. PLOS Genet. 8, e1002828.

46. Ahima, R.S., and Antwi, D.A. (2008). Brain regulation of appetite and satiety. Endocrinol. Metab. Clin. North Am. 37, 811-823.

47. Rajan, A., and Perrimon, N. (2012). Drosophila cytokine unpaired 2 regulates physiological homeostasis by remotely controlling insulin secretion. Cell 151, 123-137.

48. Friedman, J.M., and Halaas, J.L. (1998). Leptin and the regulation of body weight in mammals. Nature 395, 763-770.

49. Brent, A.E., and Rajan, A. (2020). Insulin and Leptin/Upd2 Exert Opposing Influences on Synapse Number in Fat-Sensing Neurons. Cell Metab. 32, 786-800.e7.

50. Huang, R., Song, T., Su, H., Lai, Z., Qin, W., Tian, Y., Dong, X., and Wang, L. (2020). High-fat diet enhances starvation-induced hyperactivity via sensitizing hunger-sensing neurons in Drosophila. Elife 9, e53103.

51. Brankatschk, M., Dunst, S., Nemetschke, L., and Eaton, S. (2014). Delivery of circulating lipoproteins to specific neurons in the Drosophila brain regulates systemic insulin signaling. Elife 3, e02862.

52. Géminard, C., Rulifson, E.J., and Léopold, P. (2009). Remote control of insulin secretion by fat cells in Drosophila. Cell Metab. 10, 199-207.

53. Hanson, A.J., Banks, W.A., Bettcher, L.F., Pepin, R., Raftery, D., and Craft, S. (2019).

Cerebrospinal fluid lipidomics: effects of an intravenous triglyceride infusion and apoE status. Metabolomics 16, 6.

54. Luong, N., Davies, C.R., Wessells, R.J., Graham, S.M., King, M.T., Veech, R., Bodmer, R., and Oldham, S.M. (2006). Activated FOXO-mediated insulin resistance is blocked by reduction of TOR activity. Cell Metab. 4, 133-142.

55. Biddinger, S.B., and Kahn, C.R. (2006). From mice to men: insights into the insulin resistance syndromes. Annu. Rev. Physiol. 68, 123-158.

56. Brankatschk, M., and Eaton, S. (2010). Lipoprotein Particles Cross the Blood-Brain Barrier 
bioRxiv preprint doi: https://doi.org/10.1101/2021.12.16.472854; this version posted December 21, 2021. The copyright holder for this preprint (which was not certified by peer review) is the author/funder, who has granted bioRxiv a license to display the preprint in perpetuity. It is made available under aCC-BY-NC-ND 4.0 International license.

in \&lt;em\&gt;Drosophila\&lt;/em\&gt; J. Neurosci. 30, 10441 LP - 10447. 